\title{
Augmented mixed finite element method for the Oseen problem: a priori and a posteriori error analysis *
}

\author{
Tomás P. Barrios ${ }^{\dagger}$ J. Manuel Cascón ${ }^{\ddagger}$ and María González ${ }^{\S}$
}

July 14, 2016

\begin{abstract}
We propose a new augmented dual-mixed method for the Oseen problem based on the pseudostress-velocity formulation. The stabilized formulation is obtained by adding to the dual-mixed approach suitable least squares terms that arise from the constitutive and equilibrium equations. We prove that for appropriate values of the stabilization parameters, the new variational formulation and the corresponding Galerkin scheme are well-posed, and a Céa estimate holds for any finite element subspaces. We also provide the rate of convergence when each row of the pseudostress is approximated by Raviart-Thomas or Brezzi-Douglas-Marini elements and the velocity is approximated by continuous piecewise polynomials. Moreover, we derive a simple a posteriori error estimator of residual type that consists of two residual terms and prove that it is reliable and locally efficient. Finally, we include several numerical experiments that support the theoretical results.
\end{abstract}

Mathematics Subject Classifications (1991): 65N30;65N12;65N15

Key words: incompressible flow, Oseen equation, mixed finite element, stabilized finite elements, a posteriori error estimator.

\section{Introduction}

The Oseen problem can be obtained as a linearization of the stationary incompressible Navier-Stokes equations. The most popular formulation in computational incompressible Newtonian flows is the

${ }^{*}$ The research of the first author is partially supported by Dirección de Investigación of the Universidad Católica de la Santísima Concepción (Chile) and through CONICYT-Chile FONDECYT project 1160578. The research of the second author is partially supported by the Spanish Ministerio de Ciencia e Innovación Grant CGL2011-29396-C03-02. The research of the third author is partially supported by the Spanish Ministerio de Economía y Competitividad Grant MTM2013-47800-C2-1-P.

${ }^{\dagger}$ Departamento de Matemática y Física Aplicadas, Universidad Católica de la Santísima Concepción, Casilla 297, Concepción, Chile, e-mail: tomas@ucsc.cl

${ }^{\ddagger}$ Departamento de Economía e Historia Económica, Universidad de Salamanca, Salamanca, 37008, Spain, e-mail: casbar@usal.es

${ }^{\S}$ Departamento de Matemáticas, Universidade da Coruña, Campus de Elviña s/n, 15071, A Coruña, Spain, e-mail: maria.gonzalez.taboada@udc.es 
velocity-pressure formulation. The standard Galerkin finite element method presents two difficulties. First, the presence of non-physical spurious oscillations on solutions when advection dominates makes it difficult to capture boundary layers accurately. Second, the approximation spaces for the velocity and pressure must satisfy the discrete inf-sup condition in order to ensure stability.

Several stabilized finite element methods have been developed and analyzed to handle these numerical difficulties, including the well-known streamline upwind/Petrov-Galerkin (SUPG) method [12], the pressure-stabilization/Petrov-Galerkin (PSPG) method [24, 23], grad-div stabilization [16, 22, 28], RELP methods [4], Orthogonal Subscales method [15] or Local Projection Stabilization (LPS) methods [9]. In general, a stabilized finite element method adds extra terms to the Galerkin formulation, usually suitable mesh-dependent terms, which can be residual, as in the SUPG, SUPG/PSPG or RELP methods, or non-residual, as in the Orthogonal Subscales method or LPS methods. For a thorough review of different stabilized finite element methods for the Oseen problem and a critical comparison, we refer to [10].

On the other hand, for the numerical solution of the stationary incompressible Navier-Stokes equations it is very important to have a posteriori error estimates for both Stokes and Oseen equations. The incompressibility condition and the presence of a non-self-adjoint operator in the momentum equations are the main drawbacks to obtain a posteriori error estimates for the Oseen problem. The approach in [1] is based on solving local residual problems and the analysis is valid for essentially any approximation scheme. The corresponding error estimate provides an upper bound on the true error in an energy-like norm. Moreover, under appropriate assumptions, the estimator gives two-sided bounds on the actual discretization error. We should also mention [2], where structured anisotropic mesh refinement in boundary layers is analyzed for a conforming stabilized Galerkin method of SUPG/PSPG-type with equal-order interpolation of velocity and pressure.

In last years, the interest in non-Newtonian flows has increased. In this case, when the constitutive law is nonlinear, we cannot eliminate the stress in general and the use of a formulation involving the stress seems to be mandatory. However, the stress-velocity-pressure formulation involves more unknowns and the stress tensor must be symmetric. To avoid these numerical difficulties, a mixed finite element method for the stationary Stokes equations based on the pseudostress-velocity formulation was proposed and analyzed in [13]. It is important to recall that the pseudostress, which is a nonsymmetric tensor, contains more information than the stress, and physical quantities such as the stress, velocity gradient, vorticity and pressure can be algebraically expressed in terms of the pseudostress. Therefore, these quantities could be approximated through a postprocessing procedure without degrading the accuracy.

In [13], the authors showed that using Raviart-Thomas elements of index $k \geq 0$ for approximating each row of the pseudostress and discontinuous piecewise polynomials of degree $k \geq 0$ for approximating each component of the velocity yields a stable pair and quasi-optimal accuracy $\mathcal{O}\left(h^{k+1}\right)$ for sufficiently smooth solutions. The indefinite system of linear equations resulting from the discretization can be decoupled using the penalty method. Then, the penalized pseudostress system can be solved by a multigrid method, and the velocity can be calculated explicitly/locally. In the later paper [14], the method in [13] is extended to the stationary incompressible Navier-Stokes equations. The authors show, under appropriate assumptions, that both the continuous and discrete problems have branches of nonsingular solutions, and established error estimates of the mixed finite element approximations. 
The purpose of the present work is to develop an a priori and a posteriori error analysis for a stabilized mixed finite element method for the pseudostress-velocity formulation of the Oseen equation. More precisely, we present an augmented mixed finite element method to solve the Oseen equation. The need for stabilization techniques in this case arises because of the restriction in the possible choices for the pseudostress and the velocity approximation spaces prescribed by the inf-sup condition, as well as the instabilities encountered when convection is dominant. Both can be overcome by moving from the standard Galerkin method to a stabilized formulation. The method presented here is based on the addition of suitable least-squares type terms (not mesh-dependent) to the dual-mixed formulation of the Oseen equation in pseudostress and velocity. This kind of method can be viewed as an extension of the stabilized schemes introduced in [25, 7] for Darcy flow, $[17,18,19,20]$ for linear elasticity, [6] for the Brinkman problem and [21] for the convection-diffusion equation. We show that, for appropriate values of the stabilization parameters, the augmented formulation is well-posed and the corresponding Galerkin scheme is stable for any combination of continuous velocity and pseudostress interpolations. We also provide the rate of convergence when each row of the pseudostress is approximated by RaviartThomas or Brezzi-Douglas-Marini elements, and the velocity is approximated by continuous piecewise polynomials. Moreover, we derived a new a posteriori error estimator that consists of two residual terms, and show that it is reliable and locally efficient. We implemented the corresponding adaptive algorithm in two and three dimensions. Numerical experiments confirm the theoretical properties of the method and the a posteriori error estimator.

The paper is organized as follows. In Section 2 we describe the pseudostress-velocity formulation of the model problem. In Section 3 we introduce and analyze the augmented dual-mixed variational formulation. Then, in Section 4 we analyze the stabilized mixed finite element method. Section 5 is devoted to the a posteriori error analysis of the augmented mixed finite element method. Finally, numerical experiments are reported in Section 6.

Throughout this paper, we will use the standard notations for Sobolev spaces and norms. Let $\Omega$ be a bounded, connected domain in $\mathbb{R}^{d}(d=2,3)$, with a Lipschitz-continuous boundary $\Gamma$. We recall that $H(\operatorname{div}, \Omega):=\left\{\mathbf{v} \in\left[L^{2}(\Omega)\right]^{d}: \operatorname{div}(\mathbf{v}) \in L^{2}(\Omega)\right\}, H(\operatorname{div}, \Omega):=\left\{\boldsymbol{\tau} \in\left[L^{2}(\Omega)\right]^{d \times d}: \operatorname{div}(\boldsymbol{\tau}) \in\left[L^{2}(\Omega)\right]^{d}\right\}$, and we denote by $\mathbf{H}_{0}:=\left\{\boldsymbol{\tau} \in H(\operatorname{div}, \Omega): \int_{\Omega} \operatorname{tr}(\boldsymbol{\tau})=0\right\}$, where $\operatorname{tr}(\boldsymbol{\tau})$ denotes the trace of a tensor $\boldsymbol{\tau}$.

\section{A pseudostress-velocity formulation of the model problem}

Let $\Omega$ be a bounded, connected domain in $\mathbb{R}^{d}(d=2,3)$, with a Lipschitz-continuous boundary $\Gamma$. We consider the following Oseen problem: find the velocity field $\mathbf{u}$ and the pressure $p$ such that

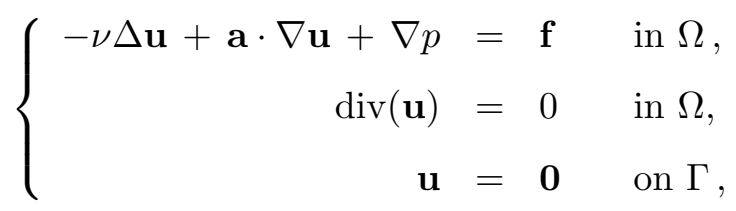

where the kinematic viscosity of the fluid $\nu>0$, the advection velocity $\mathbf{a} \neq \mathbf{0}$ and the external body force $\mathbf{f}$ are given. We assume that $\mathbf{a}$ is solenoidal in $\Omega$. 
Let $\mathbf{I}$ be the identity matrix in $\mathbb{R}^{d \times d}$ and define $\boldsymbol{\sigma}:=\nu \nabla \mathbf{u}-p \mathbf{I}$ to be the pseudostress. Then, the first equation of (1) can be written as follows:

$$
-\operatorname{div}(\boldsymbol{\sigma})+\mathbf{a} \cdot \nabla \mathbf{u}=\mathbf{f} \text { in } \Omega .
$$

On the other hand, the second equation in (1) can be stated equivalently as

$$
\operatorname{tr}(\boldsymbol{\sigma})=-d p .
$$

Thus, the pressure $p$ can be computed from the pseudostress $\sigma$. Since the pressure is determined from (1) up to an additive constant, it is usual to require that $\int_{\Omega} p=0$. Accordingly, we will impose that $\int_{\Omega} \operatorname{tr}(\boldsymbol{\sigma})=0$. Now, substituting (3) into the definition of $\boldsymbol{\sigma}$, we deduce that

$$
\boldsymbol{\sigma}^{\mathrm{d}}=\nu \nabla \mathbf{u} \quad \text { in } \Omega,
$$

where $\boldsymbol{\sigma}^{\mathrm{d}}:=\boldsymbol{\sigma}-\frac{1}{d} \operatorname{tr}(\boldsymbol{\sigma}) \mathbf{I}$ is the deviator of $\boldsymbol{\sigma}$. We remark that $\boldsymbol{\sigma}^{\mathrm{d}}$ is a trace-free tensor. Then problem (1) can be stated equivalently as follows: find $\boldsymbol{\sigma}$ and $\mathbf{u}$ such that

$$
\left\{\begin{aligned}
-\operatorname{div}(\boldsymbol{\sigma})+\mathbf{a} \cdot \nabla \mathbf{u} & =\mathbf{f} & & \text { in } \Omega, \\
\frac{1}{\nu} \boldsymbol{\sigma}^{\mathrm{d}} & =\nabla \mathbf{u} & & \text { in } \Omega, \\
\mathbf{u} & =\mathbf{0} & & \text { on } \Gamma,
\end{aligned}\right.
$$

It is easy to verify that problem (5) is equivalent to problem (1) in the sense of the following Theorem.

Theorem 1 If $(\mathbf{u}, p)$ is a solution to problem (1), then $(\boldsymbol{\sigma}, \mathbf{u})$, where $\boldsymbol{\sigma}=\nu \nabla \mathbf{u}-p \mathbf{I}$, is a solution to problem (5). Conversely, if $(\boldsymbol{\sigma}, \mathbf{u})$ is a solution to problem $(5)$, then $(\mathbf{u}, p)$, where $p=-\frac{1}{d} \operatorname{tr}(\boldsymbol{\sigma})$, is a solution to problem (1).

Next, we derive a dual-mixed variational formulation of problem (5). Multiplying the first equation of (5) by a test function $\mathbf{v}$ and integrating in $\Omega$, we obtain

$$
-\int_{\Omega} \operatorname{div}(\boldsymbol{\sigma}) \cdot \mathbf{v}+\int_{\Omega}(\mathbf{a} \cdot \nabla \mathbf{u}) \cdot \mathbf{v}=\int_{\Omega} \mathbf{f} \cdot \mathbf{v} .
$$

Then, multiplying the second equation in (5) by a test function $\tau$ and integrating in $\Omega$, we obtain, after an integration by parts in the grad-term and using the boundary condition,

$$
\frac{1}{\nu} \int_{\Omega} \boldsymbol{\sigma}^{\mathrm{d}}: \boldsymbol{\tau}^{\mathrm{d}}+\int_{\Omega} \mathbf{u} \cdot \operatorname{div}(\boldsymbol{\tau})=0
$$

where we used that $\int_{\Omega} \sigma^{\mathrm{d}}: \boldsymbol{\tau}=\int_{\Omega} \boldsymbol{\sigma}^{\mathrm{d}}: \boldsymbol{\tau}^{\mathrm{d}}$. Therefore, we have the following dual-mixed variational formulation of problem (5): find $\boldsymbol{\sigma} \in \mathbf{H}_{0}$ and $\mathbf{u} \in\left[H_{0}^{1}(\Omega)\right]^{d}$ such that

$$
\left\{\begin{array}{lll}
\frac{1}{\nu} \int_{\Omega} \boldsymbol{\sigma}^{\mathrm{d}}: \boldsymbol{\tau}^{\mathrm{d}}+\int_{\Omega} \mathbf{u} \cdot \operatorname{div}(\boldsymbol{\tau})=0, & \forall \boldsymbol{\tau} \in \mathbf{H}_{0}, \\
\int_{\Omega} \operatorname{div}(\boldsymbol{\sigma}) \cdot \mathbf{v}-\int_{\Omega}(\mathbf{a} \cdot \nabla \mathbf{u}) \cdot \mathbf{v}=-\int_{\Omega} \mathbf{f} \cdot \mathbf{v}, & \forall \mathbf{v} \in\left[H_{0}^{1}(\Omega)\right]^{d} .
\end{array}\right.
$$


Let us define the bilinear forms $a(\cdot, \cdot): \mathbf{H}_{0} \times \mathbf{H}_{0} \rightarrow \mathbb{R}, b(\cdot, \cdot):\left[H^{1}(\Omega)\right]^{d} \times \mathbf{H}_{0} \rightarrow \mathbb{R}$ and $c(\cdot, \cdot):$ $\left[H^{1}(\Omega)\right]^{d} \times\left[H^{1}(\Omega)\right]^{d} \rightarrow \mathbb{R}$ as follows:

$$
a(\boldsymbol{\sigma}, \boldsymbol{\tau}):=\frac{1}{\nu} \int_{\Omega} \boldsymbol{\sigma}^{\mathrm{d}}: \boldsymbol{\tau}^{\mathrm{d}}, \quad b(\mathbf{u}, \boldsymbol{\tau}):=\int_{\Omega} \mathbf{u} \cdot \operatorname{div}(\boldsymbol{\tau}), \quad c(\mathbf{u}, \mathbf{v}):=\int_{\Omega}(\mathbf{a} \cdot \nabla \mathbf{u}) \cdot \mathbf{v}
$$

for any $\boldsymbol{\sigma}, \boldsymbol{\tau} \in \mathbf{H}_{0}$ and $\mathbf{u}, \mathbf{v} \in\left[H^{1}(\Omega)\right]^{d}$. We also define the linear functional $l:\left[L^{2}(\Omega)\right]^{d} \rightarrow \mathbb{R}$ by $l(\mathbf{v}):=-\int_{\Omega} \mathbf{f} \cdot \mathbf{v}, \forall \mathbf{v} \in\left[L^{2}(\Omega)\right]^{d}$.

Then, the dual-mixed variational formulation (6) can be posed in the generalized saddle-point form:

$$
\left\{\begin{array}{rlrl}
a(\boldsymbol{\sigma}, \boldsymbol{\tau})+b(\mathbf{u}, \boldsymbol{\tau})=0, & & \forall \boldsymbol{\tau} \in \mathbf{H}_{0}, \\
b(\mathbf{v}, \boldsymbol{\sigma})-c(\mathbf{u}, \mathbf{v})=l(\mathbf{v}), & \forall \mathbf{v} \in\left[H_{0}^{1}(\Omega)\right]^{d},
\end{array}\right.
$$

where we remark that the bilinear form $c(\cdot, \cdot)$ is not symmetric.

According to [11], to ensure that problem (7) has a unique solution, we require, among other conditions, that the bilinear form $a(\cdot, \cdot)$ be coercive on $\mathbf{H}_{0}$. However, it is well-known that $a(\cdot, \cdot)$ is coercive in the divergence free subspace of $\mathbf{H}_{0}$ (see, for instance, the proof of Theorem 2.3 in [13]) but not on $\mathbf{H}_{0}$. We also require that the bilinear form $b(\cdot, \cdot)$ satisfies an inf-sup condition in $\mathbf{H}_{0} \times\left[H_{0}^{1}(\Omega)\right]^{d}$. These facts motivated us to consider an augmented formulation of problem (5).

\section{Augmented dual-mixed variational formulation}

In this section, we introduce an augmented variational formulation of problem (5) and show that it is well-posed for appropriate values of the stabilization parameters. We follow ideas from [25] (see also $[7,17,20,6])$ and subtract the second equation in (7) from the first one and then, add the following least-squares type terms, that arise from the equilibrium and constitutive equations in (5):

$$
\kappa_{1} \int_{\Omega}(\operatorname{div}(\boldsymbol{\sigma})-\mathbf{a} \cdot \nabla \mathbf{u}) \cdot(\operatorname{div}(\boldsymbol{\tau})+\mathbf{a} \cdot \nabla \mathbf{v})=-\kappa_{1} \int_{\Omega} \mathbf{f} \cdot(\operatorname{div}(\boldsymbol{\tau})+\mathbf{a} \cdot \nabla \mathbf{v})
$$

and

$$
\kappa_{2} \int_{\Omega}\left(\nabla \mathbf{u}-\frac{1}{\nu} \boldsymbol{\sigma}^{\mathrm{d}}\right):\left(\nabla \mathbf{v}+\frac{1}{\nu} \boldsymbol{\tau}^{\mathrm{d}}\right)=0
$$

where $(\boldsymbol{\sigma}, \mathbf{u}) \in \mathbf{H}_{0} \times\left[H_{0}^{1}(\Omega)\right]^{d}$ is a solution of $(5)$ and $(\boldsymbol{\tau}, \mathbf{v}) \in \mathbf{H}_{0} \times\left[H_{0}^{1}(\Omega)\right]^{d}$ is a test function. The parameters $\kappa_{1}$ and $\kappa_{2}$ are positive constants to be chosen so that the augmented bilinear form

$$
\begin{aligned}
& A((\boldsymbol{\sigma}, \mathbf{u}),(\boldsymbol{\tau}, \mathbf{v})):=\frac{1}{\nu} \int_{\Omega} \boldsymbol{\sigma}^{\mathrm{d}}: \boldsymbol{\tau}^{\mathrm{d}}+\int_{\Omega} \mathbf{u} \cdot \operatorname{div}(\boldsymbol{\tau})-\int_{\Omega} \operatorname{div}(\boldsymbol{\sigma}) \cdot \mathbf{v}+\int_{\Omega}(\mathbf{a} \cdot \nabla \mathbf{u}) \cdot \mathbf{v} \\
& +\kappa_{1} \int_{\Omega}(\operatorname{div}(\boldsymbol{\sigma})-\mathbf{a} \cdot \nabla \mathbf{u}) \cdot(\operatorname{div}(\boldsymbol{\tau})+\mathbf{a} \cdot \nabla \mathbf{v})+\kappa_{2} \int_{\Omega}\left(\nabla \mathbf{u}-\frac{1}{\nu} \boldsymbol{\sigma}^{\mathrm{d}}\right):\left(\nabla \mathbf{v}+\frac{1}{\nu} \boldsymbol{\tau}^{\mathrm{d}}\right)
\end{aligned}
$$

be coercive in the whole space $\mathbf{H}_{0} \times\left[H_{0}^{1}(\Omega)\right]^{d}$. 
Let us define the linear functional $F: \mathbf{H}_{0} \times\left[H_{0}^{1}(\Omega)\right]^{d} \rightarrow \mathbb{R}$ by

$$
F(\boldsymbol{\tau}, \mathbf{v}):=\int_{\Omega} \mathbf{f} \cdot \mathbf{v}-\kappa_{1} \int_{\Omega} \mathbf{f} \cdot(\operatorname{div}(\boldsymbol{\tau})+\mathbf{a} \cdot \nabla \mathbf{v}), \quad \forall(\boldsymbol{\tau}, \mathbf{v}) \in \mathbf{H}_{0} \times\left[H_{0}^{1}(\Omega)\right]^{d} .
$$

Then, the augmented variational formulation of problem (5) reads: find $(\boldsymbol{\sigma}, \mathbf{u}) \in \mathbf{H}_{0} \times\left[H_{0}^{1}(\Omega)\right]^{d}$ such that

$$
A((\boldsymbol{\sigma}, \mathbf{u}),(\boldsymbol{\tau}, \mathbf{v}))=F(\boldsymbol{\tau}, \mathbf{v}), \quad \forall(\boldsymbol{\tau}, \mathbf{v}) \in \mathbf{H}_{0} \times\left[H_{0}^{1}(\Omega)\right]^{d} .
$$

In what follows, we assume that $\mathbf{a} \in\left[L^{\infty}(\Omega)\right]^{d}$ and $\mathbf{f} \in\left[L^{2}(\Omega)\right]^{d}$.

Next we recall the following Lemma, that will be used to prove the coercivity of $A(\cdot, \cdot)$ in $\mathbf{H}_{0} \times$ $\left[H_{0}^{1}(\Omega)\right]^{d}$.

Lemma 1 There exists a positive constant, $c_{1}$, which depends only on $\Omega$, such that

$$
c_{1}\|\boldsymbol{\tau}\|_{\left[L^{2}(\Omega)\right]^{d \times d}}^{2} \leq\left\|\boldsymbol{\tau}^{\mathrm{d}}\right\|_{\left[L^{2}(\Omega)\right]^{d \times d}}^{2}+\|\operatorname{div}(\boldsymbol{\tau})\|_{\left[L^{2}(\Omega)\right]^{d}}^{2}, \quad \forall \boldsymbol{\tau} \in \mathbf{H}_{0} .
$$

Proof. See Lemma 3.1 in [3] or Proposition 3.1, Chapter IV of [11].

In the following Lemma, we prove the coercivity of $A(\cdot, \cdot)$ in $\mathbf{H}_{0} \times\left[H_{0}^{1}(\Omega)\right]^{d}$ under some conditions on the stabilization parameters.

Lemma 2 Assume that

$$
0<\kappa_{1}<\frac{\kappa_{2}}{d\|\mathbf{a}\|_{\left[L^{\infty}(\Omega)\right]^{d}}^{2}} \quad \text { and } \quad 0<\kappa_{2}<\nu .
$$

Then, there exists $C_{\mathrm{ell}}>0$ such that

$$
A((\boldsymbol{\tau}, \mathbf{v}),(\boldsymbol{\tau}, \mathbf{v})) \geq C_{\mathrm{ell}}\|(\boldsymbol{\tau}, \mathbf{v})\|_{\mathbf{H}_{0} \times\left[H_{0}^{1}(\Omega)\right]^{d}}^{2}, \quad \forall(\boldsymbol{\tau}, \mathbf{v}) \in \mathbf{H}_{0} \times\left[H_{0}^{1}(\Omega)\right]^{d} .
$$

Proof. Let $(\boldsymbol{\tau}, \mathbf{v}) \in \mathbf{H}_{0} \times\left[H_{0}^{1}(\Omega)\right]^{d}$. Then, using the definition of $A(\cdot, \cdot)$, we have

$$
\begin{aligned}
A((\boldsymbol{\tau}, \mathbf{v}),(\boldsymbol{\tau}, \mathbf{v})) & \left.=\frac{1}{\nu}\left(1-\frac{1}{\nu} \kappa_{2}\right)\left\|\boldsymbol{\tau}^{\mathrm{d}}\right\|_{\left[L^{2}(\Omega)\right]}^{2}\right]^{d \times d}+\int_{\Omega}(\mathbf{a} \cdot \nabla \mathbf{v}) \cdot \mathbf{v}+\kappa_{1}\|\operatorname{div}(\boldsymbol{\tau})\|_{\left[L^{2}(\Omega)\right]^{d}}^{2} \\
& \left.-\kappa_{1}\|\mathbf{a} \cdot \nabla \mathbf{v}\|_{\left[L^{2}(\Omega)\right]^{d}}^{2}+\kappa_{2}\|\nabla \mathbf{v}\|_{\left[L^{2}(\Omega)\right]}^{2}\right]^{d \times d}
\end{aligned}
$$

We remark that, since $\mathbf{a}$ is solenoidal, then $\int_{\Omega}(\mathbf{a} \cdot \nabla \mathbf{v}) \cdot \mathbf{v}=0$. On the other hand, it is easy to see that

$$
\|\mathbf{a} \cdot \nabla \mathbf{v}\|_{\left[L^{2}(\Omega)\right]^{d}}^{2} \leq d\|\mathbf{a}\|_{\left[L^{\infty}(\Omega)\right]^{d}}^{2}\|\nabla \mathbf{v}\|_{\left[L^{2}(\Omega)\right]^{d \times d}}^{2}
$$

Therefore,

$$
\begin{aligned}
A((\boldsymbol{\tau}, \mathbf{v}),(\boldsymbol{\tau}, \mathbf{v})) & \geq \frac{1}{\nu}\left(1-\frac{\kappa_{2}}{\nu}\right)\left\|\boldsymbol{\tau}^{\mathrm{d}}\right\|_{\left[L^{2}(\Omega)\right]^{d \times d}}^{2}+\kappa_{1}\|\operatorname{div}(\boldsymbol{\tau})\|_{\left[L^{2}(\Omega)\right]^{d}}^{2} \\
& +\left(\kappa_{2}-\kappa_{1} d\|\mathbf{a}\|_{\left[L^{\infty}(\Omega)\right]^{d}}^{2}\|\nabla \mathbf{v}\|_{\left[L^{2}(\Omega)\right]^{d \times d}}^{2}\right.
\end{aligned}
$$


Then, by applying Lemma 1 and Poincaré inequality, the ellipticity of $A(\cdot, \cdot)$ follows for the feasible values of $\kappa_{1}$ and $\kappa_{2}$, with

$$
C_{\mathrm{ell}}=\min \left(\frac{1}{\nu}\left(1-\frac{\kappa_{2}}{\nu}\right) c_{1}, \frac{\kappa_{1}}{2} c_{1}, \frac{\kappa_{1}}{2},\left(\kappa_{2}-\kappa_{1} d\|\mathbf{a}\|_{\left[L^{\infty}(\Omega)\right]^{d}}^{2}\right) C_{\Omega}\right),
$$

where $C_{\Omega}$ is the Poincaré constant.

Remark. We assumed that $\mathbf{a} \neq \mathbf{0}$. In case $\mathbf{a}=\mathbf{0}$, we would be dealing with the Stokes problem. In this case,

$$
C_{\mathrm{ell}}=\min \left(\frac{1}{\nu}\left(1-\frac{\kappa_{2}}{\nu}\right) c_{1}, \frac{\kappa_{1}}{2} c_{1}, \frac{\kappa_{1}}{2}, \kappa_{2} C_{\Omega}\right),
$$

so that the values of the stabilization parameters that guarantee the coerciveness of the augmented bilinear form $A(\cdot, \cdot)$ are

$$
\kappa_{1}>0, \quad \kappa_{2} \in(0, \nu) .
$$

Theorem 2 Assume the hypotheses of Lemma 2. Then, problem (10) has a unique solution $(\boldsymbol{\sigma}, \mathbf{u}) \in$ $\mathbf{H}_{0} \times\left[H_{0}^{1}(\Omega)\right]^{d}$ and

$$
\|(\boldsymbol{\sigma}, \mathbf{u})\|_{\mathbf{H}_{0} \times\left[H_{0}^{1}(\Omega)\right]^{d}} \leq C_{\mathrm{ell}}^{-1}\left(1+\kappa_{1}\left(1+\sqrt{d}\|\mathbf{a}\|_{\left[L^{\infty}(\Omega)\right]^{d}}\right)\right)\|\mathbf{f}\|_{\left[L^{2}(\Omega)\right]^{d}} .
$$

Proof. From Lemma 2, the continuous bilinear form $A(\cdot, \cdot)$ is elliptic in $\mathbf{H}_{0} \times\left[H_{0}^{1}(\Omega)\right]^{d}$. On the other hand, the linear functional $F$ is continuous in $\mathbf{H}_{0} \times\left[H_{0}^{1}(\Omega)\right]^{d}$, with

$$
|F(\boldsymbol{\tau}, \mathbf{v})| \leq\left(1+\kappa_{1}\left(1+\sqrt{d}\|\mathbf{a}\|_{\left[L^{\infty}(\Omega)\right]^{d}}\right)\right)\|\mathbf{f}\|_{\left[L^{2}(\Omega)\right]^{d}}\|(\boldsymbol{\tau}, \mathbf{v})\|_{\mathbf{H}_{0} \times\left[H_{0}^{1}(\Omega)\right]^{d}} .
$$

The result follows by applying the Lax-Milgram Lemma.

Remark. In order to maximize the ellipticity constant, $C_{\mathrm{ell}}$, we choose the stabilization parameters $\kappa_{1}$ and $\kappa_{2}$ to be:

$$
\tilde{\kappa}_{1}=\frac{\nu}{\frac{c_{1}}{2 C_{\Omega}}+\frac{\nu^{2}}{2}+d\|\mathbf{a}\|_{\left[L^{\infty}(\Omega)\right]^{d}}^{2}}, \quad \tilde{\kappa}_{2}=\frac{\nu\left(\frac{c_{1}}{2 C_{\Omega}}+d\|\mathbf{a}\|_{\left[L^{\infty}(\Omega)\right]^{d}}^{2}\right)}{\frac{c_{1}}{2 C_{\Omega}}+\frac{\nu^{2}}{2}+d\|\mathbf{a}\|_{\left[L^{\infty}(\Omega)\right]^{d}}^{2}} .
$$

This choice characterizes the ellipticity constant as a function of the physical parameters as:

$$
C_{\mathrm{ell}}(\nu, \mathbf{a})=\frac{\tilde{\kappa}_{1}}{2} c_{1}=\frac{\nu c_{1}}{\frac{c_{1}}{C_{\Omega}}+\nu^{2}+2 d\|\mathbf{a}\|_{\left[L^{\infty}(\Omega)\right]^{d}}^{2}} .
$$

Thus, when convection is dominant $\left(\nu<<\|\mathbf{a}\|_{\left[L^{\infty}(\Omega)\right]^{d}}\right)$, we would have $c \nu\|\mathbf{a}\|_{\left[L^{\infty}(\Omega)\right]^{d}}^{-2} \leq C_{\mathrm{ell}} \leq$ $C\|\mathbf{a}\|_{\left[L^{\infty}(\Omega)\right]^{d}}^{-1}$. Moreover, the continuous dependence constant in Theorem 2 will be of $\mathcal{O}\left(\nu^{-1}\|\mathbf{a}\|_{\left[L^{\infty}(\Omega)\right]^{d}}^{2}+\right.$ $\|\mathbf{a}\|_{\left.\left[L^{\infty}(\Omega)\right]^{d}\right)}$. 


\section{Augmented mixed finite element method}

In what follows, we assume that $\Omega$ is a polygonal $(d=2)$ or polyhedral $(d=3)$ domain. Let $\left\{\mathcal{T}_{h}\right\}_{h>0}$ be a family of shape-regular meshes of $\bar{\Omega}$ made up of triangles $(d=2)$ or tetrahedra $(d=3)$. We denote by $h_{T}$ the diameter of an element $T \in \mathcal{T}_{h}$ and define $h:=\max _{T \in \mathcal{T}_{h}} h_{T}$. Let $\mathbf{H}_{0, h}$ and $V_{h}$ be any finite element subspaces of $\mathbf{H}_{0}$ and $\left[H_{0}^{1}(\Omega)\right]^{d}$, respectively. Then, the Galerkin scheme associated to problem (10) reads: find $\left(\boldsymbol{\sigma}_{h}, \mathbf{u}_{h}\right) \in \mathbf{H}_{0, h} \times V_{h}$ such that

$$
A\left(\left(\boldsymbol{\sigma}_{h}, \mathbf{u}_{h}\right),\left(\boldsymbol{\tau}_{h}, \mathbf{v}_{h}\right)\right)=F\left(\boldsymbol{\tau}_{h}, \mathbf{v}_{h}\right), \quad \forall\left(\boldsymbol{\tau}_{h}, \mathbf{v}_{h}\right) \in \mathbf{H}_{0, h} \times V_{h} .
$$

Under the hypotheses of Lemma 2, the bilinear form $A(\cdot, \cdot)$ is coercive in $\mathbf{H}_{0} \times\left[H_{0}^{1}(\Omega)\right]^{d}$, and hence, in any finite element subspace $\mathbf{H}_{0, h} \times V_{h}$ of $\mathbf{H}_{0} \times\left[H_{0}^{1}(\Omega)\right]^{d}$. Therefore, problem (13) has a unique solution $\left(\boldsymbol{\sigma}_{h}, \mathbf{u}_{h}\right) \in \mathbf{H}_{0, h} \times V_{h}$. Moreover, there exists a constant $C_{\text {Cea }}>0$, independent of $h$, such that

$$
\left\|\left(\boldsymbol{\sigma}-\boldsymbol{\sigma}_{h}, \mathbf{u}-\mathbf{u}_{h}\right)\right\|_{\mathbf{H}_{0} \times\left[H_{0}^{1}(\Omega)\right]^{d}} \leq C_{\mathrm{Cea}} \inf _{\left(\boldsymbol{\tau}_{h}, \mathbf{v}_{h}\right) \in \mathbf{H}_{0, h} \times V_{h}}\left\|\left(\boldsymbol{\sigma}-\boldsymbol{\tau}_{h}, \mathbf{u}-\mathbf{v}_{h}\right)\right\|_{\mathbf{H}_{0} \times\left[H_{0}^{1}(\Omega)\right]^{d}},
$$

where for the values $\tilde{\kappa}_{1}$ and $\tilde{\kappa}_{2}$ defined in (11), $C_{\text {Cea }}=\mathcal{O}\left(\nu^{-1}\|\mathbf{a}\|_{\left[L^{\infty}(\Omega)\right]^{d}}^{3}\right)$ if $\nu<<\|\mathbf{a}\|_{\left[L^{\infty}(\Omega)\right]^{d}}$.

In order to establish a rate of convergence result, we consider specific finite element subspaces $\mathbf{H}_{0, h}$ and $V_{h}$. Hereafter, given $T \in \mathcal{T}_{h}$ and an integer $l \geq 0$, we denote by $\mathcal{P}_{l}(T)$ the space of polynomials of total degree at most $l$ on $T$ and, given an integer $r \geq 0$, we denote by $\mathcal{R} \mathcal{T}_{r}(T)$ the local Raviart-Thomas space of order $r+1$ (cf. [26]),

$$
\mathcal{R} \mathcal{T}_{r}(T):=\left[\mathcal{P}_{r}(T)\right]^{d} \oplus[\mathbf{x}] \mathcal{P}_{r}(T) \subset\left[\mathcal{P}_{r+1}(T)\right]^{d},
$$

where $\mathbf{x}$ is a generic vector of $\mathbb{R}^{d}$.

Let $r \geq 0$ and $m \geq 1$. Then, we let $\mathbf{H}_{0, h}$ be either

$$
\mathbf{H}_{0, h}:=\left[\mathcal{R} \mathcal{T}_{r}^{\mathrm{t}}\right]^{d}=\left\{\boldsymbol{\tau}_{h} \in \mathbf{H}_{0}:\left.\boldsymbol{\tau}_{h}\right|_{T} \in\left[\mathcal{R} \mathcal{T}_{r}(T)^{\mathrm{t}}\right]^{d}, \quad \forall T \in \mathcal{T}_{h}\right\}
$$

or

$$
\mathbf{H}_{0, h}:=\left[\mathcal{B D} \mathcal{M}_{r+1}^{\mathrm{t}}\right]^{d}=\left\{\boldsymbol{\tau}_{h} \in \mathbf{H}_{0}:\left.\left(\boldsymbol{\tau}_{h}\right)_{i}\right|_{T} \in\left[\mathcal{P}_{r+1}(T)\right]^{d}, \quad i=1, \ldots, d, \quad \forall T \in \mathcal{T}_{h}\right\},
$$

where $\left(\boldsymbol{\tau}_{h}\right)_{i}$ denotes the $i$-th row of $\boldsymbol{\tau}_{h}$, and define

$$
V_{h}:=\left[\mathcal{L}_{m}\right]^{d}=\left\{\mathbf{v}_{h} \in\left[\mathcal{C}(\bar{\Omega}) \cap H_{0}^{1}(\Omega)\right]^{d}:\left.\mathbf{v}_{h}\right|_{T} \in\left[\mathcal{P}_{m}(T)\right]^{d}, \quad \forall T \in \mathcal{T}_{h}\right\} .
$$

The corresponding rate of convergence is given in the next theorem.

Theorem 3 Assume $\boldsymbol{\sigma} \in\left[H^{t}(\Omega)\right]^{d \times d}, \operatorname{div}(\boldsymbol{\sigma}) \in\left[H^{t}(\Omega)\right]^{d}$ and $\mathbf{u} \in\left[H^{t+1}(\Omega)\right]^{d}$. Then, under the assumptions of Lemma 2, there exists $C=\mathcal{O}\left(C_{\mathrm{Cea}}\right)>0$, independent of $h$, such that

$$
\left\|\left(\boldsymbol{\sigma}-\boldsymbol{\sigma}_{h}, \mathbf{u}-\mathbf{u}_{h}\right)\right\|_{\mathbf{H}_{0} \times\left[H_{0}^{1}(\Omega)\right]^{d}} \leq C h^{\min \{t, m, r+1\}}\left(\|\boldsymbol{\sigma}\|_{\left[H^{t}(\Omega)\right]^{d \times d}}+\|\operatorname{div}(\boldsymbol{\sigma})\|_{\left[H^{t}(\Omega)\right]^{d}}+\|\mathbf{u}\|_{\left[H^{t+1}(\Omega)\right]^{d}}\right) .
$$


Proof. It follows straightforwardly from inequality (14) and the approximation properties of the corresponding finite element subspaces.

Remark. Under the hypothesis of the previous Theorem, we have that

$$
\left\|p-p_{h}\right\|_{L^{2}(\Omega)} \leq C h^{\min \{t, m, r+1\}}\left(\|\boldsymbol{\sigma}\|_{\left[H^{t}(\Omega)\right]^{d \times d}}+\|\operatorname{div}(\boldsymbol{\sigma})\|_{\left[H^{t}(\Omega)\right]^{d}}+\|\mathbf{u}\|_{\left[H^{t+1}(\Omega)\right]^{d}}\right),
$$

where we recover $p_{h}=-\frac{1}{d} \operatorname{tr}\left(\boldsymbol{\sigma}_{h}\right)$ and $C=\mathcal{O}\left(C_{\text {Cea }}\right)$.

Remark. If we approximate $\boldsymbol{\sigma}$ by continuous piecewise polynomials, that is, if we choose

$$
\mathbf{H}_{0, h}=\left\{\boldsymbol{\tau}_{h} \in \mathbf{H}_{0} \cap[\mathcal{C}(\bar{\Omega})]^{d \times d}:\left.\left(\boldsymbol{\tau}_{h}\right)\right|_{T} \in\left[\mathcal{P}_{r}(T)\right]^{d \times d}, \quad \forall T \in \mathcal{T}_{h}\right\},
$$

we will obtain the corresponding error estimate

$$
\left\|\left(\boldsymbol{\sigma}-\boldsymbol{\sigma}_{h}, \mathbf{u}-\mathbf{u}_{h}\right)\right\|_{\mathbf{H}_{0} \times\left[H_{0}^{1}(\Omega)\right]^{d}} \leq C h^{\min \{t, m, r\}}\left(\|\boldsymbol{\sigma}\|_{\left[H^{t+1}(\Omega)\right]^{d \times d}}+\|\mathbf{u}\|_{\left[H^{t+1}(\Omega)\right]^{d}}\right) .
$$

for $\boldsymbol{\sigma} \in\left[H^{t+1}(\Omega)\right]^{d \times d}$ and $\mathbf{u} \in\left[H^{t+1}(\Omega)\right]^{d}$, with $C=\mathcal{O}\left(C_{\text {Cea }}\right)$. Notice that this result requires more regularity on $\sigma$.

\section{$5 \quad$ A posteriori error analysis}

In this section, we develop a residual-based a posteriori error analysis of the augmented mixed finite element method (13). That analysis is similar to the one presented in [5] and [7] for the linear elasticity and the Darcy problems, respectively. We derive a simple a posteriori error estimator that consists of two residual terms and show that it is reliable and locally efficient. In what follows, we assume that the stabilization parameters, $\kappa_{1}$ and $\kappa_{2}$, satisfy the hypotheses of Lemma 2.

Let $\mathbf{H}_{0, h}$ and $V_{h}$ be any finite element subspaces of $\mathbf{H}_{0}$ and $\left[H_{0}^{1}(\Omega)\right]^{d}$, respectively, and let $(\boldsymbol{\sigma}, \mathbf{u}) \in$ $\mathbf{H}_{0} \times\left[H_{0}^{1}(\Omega)\right]^{d}$ and $\left(\boldsymbol{\sigma}_{h}, \mathbf{u}_{h}\right) \in \mathbf{H}_{0, h} \times V_{h}$ be the unique solutions to problems (10) and (13), respectively. Then, we define the residual

$$
R_{h}(\boldsymbol{\tau}, \mathbf{v})=F(\boldsymbol{\tau}, \mathbf{v})-A\left(\left(\boldsymbol{\sigma}_{h}, \mathbf{u}_{h}\right),(\boldsymbol{\tau}, \mathbf{v})\right), \quad \forall(\boldsymbol{\tau}, \mathbf{v}) \in \mathbf{H}_{0} \times\left[H_{0}^{1}(\Omega)\right]^{d} .
$$

Applying the coercivity of the bilinear form $A(\cdot, \cdot)$ to the error, $\left(\boldsymbol{\sigma}-\boldsymbol{\sigma}_{h}, \mathbf{u}-\mathbf{u}_{h}\right) \in \mathbf{H}_{0} \times\left[H_{0}^{1}(\Omega)\right]^{d}$, and using the definition of the residual (16), we deduce that

$$
\begin{aligned}
\left\|\left(\boldsymbol{\sigma}-\boldsymbol{\sigma}_{h}, \mathbf{u}-\mathbf{u}_{h}\right)\right\|_{\mathbf{H}_{0} \times\left[H_{0}^{1}(\Omega)\right]^{d}} & \leq C_{\mathrm{ell}}^{-1} \frac{A\left(\left(\boldsymbol{\sigma}-\boldsymbol{\sigma}_{h}, \mathbf{u}-\mathbf{u}_{h}\right),\left(\boldsymbol{\sigma}-\boldsymbol{\sigma}_{h}, \mathbf{u}-\mathbf{u}_{h}\right)\right)}{\left\|\left(\boldsymbol{\sigma}-\boldsymbol{\sigma}_{h}, \mathbf{u}-\mathbf{u}_{h}\right)\right\|_{\mathbf{H}_{0} \times\left[H_{0}^{1}(\Omega)\right]^{d}}} \\
& \leq C_{\mathrm{ell}}^{-1} \sup _{\substack{(\boldsymbol{\tau}, \mathbf{v}) \in \mathbf{H}_{0} \times\left[H_{0}^{1}(\Omega)\right]^{d} \\
(\boldsymbol{\tau}, \mathbf{v}) \neq(0,0)}} \frac{A\left(\left(\boldsymbol{\sigma}-\boldsymbol{\sigma}_{h}, \mathbf{u}-\mathbf{u}_{h}\right),(\boldsymbol{\tau}, \mathbf{v})\right)}{\|(\boldsymbol{\tau}, \mathbf{v})\|_{\mathbf{H}_{0} \times\left[H_{0}^{1}(\Omega)\right]^{d}}} \\
& =C_{\text {ell }}^{-1} \sup _{\substack{\boldsymbol{\tau}, \mathbf{v}) \in \mathbf{H}_{0} \times\left[H_{0}^{1}(\Omega)\right]^{d} \\
(\boldsymbol{\tau}, \mathbf{v}) \neq(0,0)}} \frac{R_{h}(\boldsymbol{\tau}, \mathbf{v})}{\|(\boldsymbol{\tau}, \mathbf{v})\|_{\mathbf{H}_{0} \times\left[H_{0}^{1}(\Omega)\right]^{d}}}
\end{aligned}
$$

In the next Lemma, we obtain an upper bound for the residual. 
Lemma 3 There exists a positive constant $C$, independent of $h$, such that

$$
\sup _{\substack{(\boldsymbol{\tau}, \mathbf{v}) \in \mathbf{H}_{0} \times\left[H_{0}^{1}(\Omega)\right]^{d} \\(\boldsymbol{\tau}, \mathbf{v}) \neq(0,0)}} \frac{R_{h}(\boldsymbol{\tau}, \mathbf{v})}{\|(\boldsymbol{\tau}, \mathbf{v})\|_{\mathbf{H}_{0} \times\left[H_{0}^{1}(\Omega)\right]^{d}}} \leq C\left(\left\|\mathbf{f}+\operatorname{div}\left(\boldsymbol{\sigma}_{h}\right)-\mathbf{a} \cdot \nabla \mathbf{u}_{h}\right\|_{\left[L^{2}(\Omega)\right]^{d}}+\left\|\nabla \mathbf{u}_{h}-\frac{1}{\nu} \boldsymbol{\sigma}_{h}^{\mathrm{d}}\right\|_{\left[L^{2}(\Omega)\right]^{d \times d}}\right) .
$$

Proof. Using the definitions of the linear functional $F$ and the bilinear form $A(\cdot, \cdot)$, we have

$$
R_{h}(\boldsymbol{\tau}, \mathbf{v})=R_{1}(\boldsymbol{\tau})+R_{2}(\mathbf{v}), \quad \forall \boldsymbol{\tau} \in \mathbf{H}_{0}, \quad \forall \mathbf{v} \in\left[H_{0}^{1}(\Omega)\right]^{d},
$$

where $R_{1}: \mathbf{H}_{0} \rightarrow \mathbb{R}$ and $R_{2}:\left[H_{0}^{1}(\Omega)\right]^{d} \rightarrow \mathbb{R}$ are defined by

$$
\begin{gathered}
R_{1}(\boldsymbol{\tau}):=-\kappa_{1} \int_{\Omega} \mathbf{f} \cdot \operatorname{div}(\boldsymbol{\tau})-\int_{\Omega} \mathbf{u}_{h} \cdot \operatorname{div}(\boldsymbol{\tau})-\kappa_{1} \int_{\Omega}\left(\operatorname{div}\left(\boldsymbol{\sigma}_{h}\right)-\mathbf{a} \cdot \nabla \mathbf{u}_{h}\right) \cdot \operatorname{div}(\boldsymbol{\tau}) \\
-\frac{1}{\nu} \int_{\Omega} \boldsymbol{\sigma}_{h}^{\mathrm{d}}: \boldsymbol{\tau}^{\mathrm{d}}-\frac{\kappa_{2}}{\nu} \int_{\Omega}\left(\nabla \mathbf{u}_{h}-\frac{1}{\nu} \boldsymbol{\sigma}_{h}^{\mathrm{d}}\right): \boldsymbol{\tau}^{\mathrm{d}}, \\
R_{2}(\mathbf{v}):=\int_{\Omega}\left(\mathbf{f}+\operatorname{div}\left(\boldsymbol{\sigma}_{h}\right)-\mathbf{a} \cdot \nabla \mathbf{u}_{h}\right) \cdot \mathbf{v}-\kappa_{1} \int_{\Omega}\left(\mathbf{f}+\operatorname{div}\left(\boldsymbol{\sigma}_{h}\right)-\mathbf{a} \cdot \nabla \mathbf{u}_{h}\right) \cdot(\mathbf{a} \cdot \nabla \mathbf{v})-\kappa_{2} \int_{\Omega}\left(\nabla \mathbf{u}_{h}-\frac{1}{\nu} \boldsymbol{\sigma}_{h}^{\mathrm{d}}\right): \nabla \mathbf{v} .
\end{gathered}
$$

Then, integrating by parts the second term in $R_{1}$ and using the boundary condition, we deduce that

$$
R_{1}(\boldsymbol{\tau})=-\kappa_{1} \int_{\Omega}\left(\mathbf{f}+\operatorname{div}\left(\boldsymbol{\sigma}_{h}\right)-\mathbf{a} \cdot \nabla \mathbf{u}_{h}\right) \cdot \operatorname{div}(\boldsymbol{\tau})+\int_{\Omega}\left(\nabla \mathbf{u}_{h}-\frac{1}{\nu} \boldsymbol{\sigma}_{h}^{\mathrm{d}}\right): \boldsymbol{\tau}-\frac{\kappa_{2}}{\nu} \int_{\Omega}\left(\nabla \mathbf{u}_{h}-\frac{1}{\nu} \boldsymbol{\sigma}_{h}^{\mathrm{d}}\right): \boldsymbol{\tau}^{\mathrm{d}} .
$$
have

Now, applying the Cauchy-Schwarz inequality and using that $\left\|\boldsymbol{\tau}^{\mathrm{d}}\right\|_{\left[L^{2}(\Omega)\right]^{d \times d}} \leq\|\boldsymbol{\tau}\|_{\left[L^{2}(\Omega)\right]^{d \times d}}$, we

$$
\begin{aligned}
\left|R_{1}(\boldsymbol{\tau})\right| \leq \kappa_{1}\left\|\mathbf{f}+\operatorname{div}\left(\boldsymbol{\sigma}_{h}\right)-\mathbf{a} \cdot \nabla \mathbf{u}_{h}\right\|_{\left[L^{2}(\Omega)\right]^{d}}\|\operatorname{div}(\boldsymbol{\tau})\|_{\left[L^{2}(\Omega)\right]^{d}} \\
+\left(1+\frac{\kappa_{2}}{\nu}\right)\left\|\nabla \mathbf{u}_{h}-\frac{1}{\nu} \boldsymbol{\sigma}_{h}^{\mathrm{d}}\right\|_{\left[L^{2}(\Omega)\right]^{d \times d}}\|\boldsymbol{\tau}\|_{\left[L^{2}(\Omega)\right]^{d \times d}}
\end{aligned}
$$

and

$$
\begin{aligned}
\left|R_{2}(\mathbf{v})\right| & \leq\left\|\mathbf{f}+\operatorname{div}\left(\boldsymbol{\sigma}_{h}\right)-\mathbf{a} \cdot \nabla \mathbf{u}_{h}\right\|_{\left[L^{2}(\Omega)\right]^{d}}\|\mathbf{v}\|_{\left[L^{2}(\Omega)\right]^{d}} \\
& +\kappa_{1} \sqrt{d}\|\mathbf{a}\|_{\left[L^{\infty}(\Omega)\right]^{d}}\left\|\mathbf{f}+\operatorname{div}\left(\boldsymbol{\sigma}_{h}\right)-\mathbf{a} \cdot \nabla \mathbf{u}_{h}\right\|_{\left[L^{2}(\Omega)\right]^{d}}\|\nabla \mathbf{v}\|_{\left[L^{2}(\Omega)\right]^{d \times d}} \\
& +\kappa_{2}\left\|\nabla \mathbf{u}_{h}-\frac{1}{\nu} \boldsymbol{\sigma}_{h}^{\mathrm{d}}\right\|_{\left[L^{2}(\Omega)\right]^{d \times d}}\|\nabla \mathbf{v}\|_{\left[L^{2}(\Omega)\right]^{d \times d}}
\end{aligned}
$$

Therefore, the proof follows using the triangle inequality, with

$$
C=\max \left(1+\kappa_{1}\left(1+\sqrt{d}\|\mathbf{a}\|_{\left[L^{\infty}(\Omega)\right]^{d}}\right), 1+\kappa_{2}\left(1+\frac{1}{\nu}\right)\right) .
$$


Motivated by the previous result, we define the a posteriori error estimator $\theta$ as follows:

$$
\theta^{2}:=\sum_{T \in \mathcal{T}_{h}} \theta_{T}^{2}, \quad \text { with } \quad \theta_{T}^{2}:=\left\|\mathbf{f}+\operatorname{div}\left(\boldsymbol{\sigma}_{h}\right)-\mathbf{a} \cdot \nabla \mathbf{u}_{h}\right\|_{\left[L^{2}(T)\right]^{d}}^{2}+\left\|\nabla \mathbf{u}_{h}-\frac{1}{\nu} \boldsymbol{\sigma}_{h}^{\mathrm{d}}\right\|_{\left[L^{2}(T)\right]^{d \times d}}^{2} .
$$

In the next theorem we establish the equivalence between the total error and the estimator $\theta$. Inequality (19) means that the a posteriori error estimator $\theta$ is reliable, whereas inequality (20) means that $\theta$ is locally efficient.

Theorem 4 Let $(\boldsymbol{\sigma}, \mathbf{u}) \in \mathbf{H}_{0} \times\left[H_{0}^{1}(\Omega)\right]^{d}$ and $\left(\boldsymbol{\sigma}_{h}, \mathbf{u}_{h}\right) \in \mathbf{H}_{0, h} \times V_{h}$ be the unique solutions to problems (10) and (13), respectively. Then, there exists a positive constant $C_{\mathrm{rel}}$, independent of $h$, such that

$$
\left\|\left(\boldsymbol{\sigma}-\boldsymbol{\sigma}_{h}, \mathbf{u}-\mathbf{u}_{h}\right)\right\|_{\mathbf{H}_{0} \times\left[H_{0}^{1}(\Omega)\right]^{d}} \leq C_{\mathrm{rel}} \theta,
$$

and there exists a positive constant $C_{\text {eff }}$, independent of $h$ and $T$, such that

$$
C_{\text {eff }} \theta_{T} \leq\left\|\left(\boldsymbol{\sigma}-\boldsymbol{\sigma}_{h}, \mathbf{u}-\mathbf{u}_{h}\right)\right\|_{\mathbf{H}(\operatorname{div}, T) \times H^{1}(T)}, \quad \forall T \in \mathcal{T}_{h} .
$$

Proof. Using (17), Lemma 3 and the definition of $\theta$, we deduce inequality (19) with $C_{\text {rel }}:=\sqrt{2} C_{\text {ell }}^{-1} C$, with $C$ being the constant of Lemma 3. On the other hand, to prove inequality (20), we recall from (5) that $\mathbf{f}=-\operatorname{div}(\boldsymbol{\sigma})+\mathbf{a} \cdot \nabla \mathbf{u}$ and $\frac{1}{\nu} \boldsymbol{\sigma}^{\mathrm{d}}-\nabla \mathbf{u}=\mathbf{0}$ in $\Omega$. Then, using the triangle inequality

$$
\begin{aligned}
\left\|\mathbf{f}+\operatorname{div}\left(\boldsymbol{\sigma}_{h}\right)-\mathbf{a} \cdot \nabla \mathbf{u}_{h}\right\|_{\left[L^{2}(T)\right]^{d}}^{2} & =\left\|\operatorname{div}\left(\boldsymbol{\sigma}_{h}-\boldsymbol{\sigma}\right)-\mathbf{a} \cdot \nabla\left(\mathbf{u}_{h}-\mathbf{u}\right)\right\|_{\left[L^{2}(T)\right]^{d}}^{2} \\
& \leq 2\left(\left\|\operatorname{div}\left(\boldsymbol{\sigma}_{h}-\boldsymbol{\sigma}\right)\right\|_{\left[L^{2}(T)\right]^{d}}^{2}+\left\|\mathbf{a} \cdot \nabla\left(\mathbf{u}_{h}-\mathbf{u}\right)\right\|_{\left[L^{2}(T)\right]^{d}}^{2}\right) \\
& \leq 2\left(\left\|\operatorname{div}\left(\boldsymbol{\sigma}_{h}-\boldsymbol{\sigma}\right)\right\|_{\left[L^{2}(T)\right]^{d}}^{2}+d\|\mathbf{a}\|_{\left[L^{\infty}(\Omega)\right]^{d}}^{2}\left\|\nabla\left(\mathbf{u}_{h}-\mathbf{u}\right)\right\|_{\left[L^{2}(T)\right]^{d \times d}}^{2}\right),
\end{aligned}
$$

and

$$
\begin{aligned}
\left\|\nabla \mathbf{u}_{h}-\frac{1}{\nu} \boldsymbol{\sigma}_{h}^{\mathrm{d}}\right\|_{\left[L^{2}(T)\right] d \times d}^{2} & \leq 2\left(\left\|\nabla\left(\mathbf{u}_{h}-\mathbf{u}\right)\right\|_{\left[L^{2}(T)\right] d \times d}^{2}+\frac{1}{\nu^{2}}\left\|\left(\boldsymbol{\sigma}_{h}-\boldsymbol{\sigma}\right)^{\mathrm{d}}\right\|_{\left[L^{2}(T)\right]^{d \times d}}^{2}\right) \\
& \leq 2\left(\left\|\nabla\left(\mathbf{u}_{h}-\mathbf{u}\right)\right\|_{\left[L^{2}(T)\right]^{d \times d}}^{2}+\frac{1}{\nu^{2}}\left\|\boldsymbol{\sigma}_{h}-\boldsymbol{\sigma}\right\|_{\left[L^{2}(T)\right]^{d \times d}}^{2}\right) .
\end{aligned}
$$

Therefore,

$$
\theta_{T}^{2} \leq 2\left(\max \left(1, \frac{1}{\nu^{2}}\right)\left\|\boldsymbol{\sigma}_{h}-\boldsymbol{\sigma}\right\|_{\mathbf{H}(\mathbf{d i v} ; T)}^{2}+2\left(1+d\|\mathbf{a}\|_{\left[L^{\infty}(\Omega)\right]^{d}}^{2}\left\|\nabla\left(\mathbf{u}_{h}-\mathbf{u}\right)\right\|_{\left[L^{2}(T)\right]^{d \times d}}^{2}\right)\right.
$$

and the proof follows with $C_{\text {eff }}^{-1}:=\sqrt{2 \max \left(\frac{1}{\nu^{2}}, 1+d\|\mathbf{a}\|_{\left[L^{\infty}(\Omega)\right]^{d}}^{2}\right)}$.

Remark. From the previous analysis, we have that if $\nu<<\|\mathbf{a}\|_{\left[L^{\infty}(\Omega)\right]^{d}}$, then the efficiency constant is $C_{\text {eff }}^{-1}=\mathcal{O}\left(\max \left(\frac{1}{\nu},\|\mathbf{a}\|_{\left[L^{\infty}(\Omega)\right]^{d}}\right)\right)$. We notice that this constant is independent of the choice of the stabilization parameters $\kappa_{1}$ and $\kappa_{2}$. If $\nu<<1 \leq\|\mathbf{a}\|_{\left[L^{\infty}(\Omega)\right]^{d}}$, for the choice (11), the reliability constant is $C_{\mathrm{rel}}=\mathcal{O}\left(C_{\mathrm{ell}}^{-1}\right)=\mathcal{O}\left(\nu\|\mathbf{a}\|_{\left[L^{\infty}(\Omega)\right]^{d}}^{-2}\right)$. 


\section{$6 \quad$ Numerical experiments}

In this section, we present some numerical experiments that illustrate the performance of the augmented scheme (13) and confirm the properties of the a posteriori error estimator $\theta$ defined in (18).

In order to implement the mean zero condition for the traces of functions in the space $\mathbf{H}_{0, h}$, we introduce, as described in [8], a Lagrange multiplier $\left(\varphi_{h} \in \mathbb{R}\right.$ below). That is, instead of (13), we consider the equivalent problem: find $\left(\boldsymbol{\sigma}_{h}, \mathbf{u}_{h}, \varphi_{h}\right) \in \mathbf{H}_{h} \times V_{h} \times \mathbb{R}$ such that

$$
\left\{\begin{aligned}
A\left(\left(\boldsymbol{\sigma}_{h}, \mathbf{u}_{h}\right),\left(\boldsymbol{\tau}_{h}, \mathbf{v}_{h}\right)\right)+\varphi_{h} \int_{\Omega} \operatorname{tr}\left(\boldsymbol{\tau}_{h}\right) & =F\left(\boldsymbol{\tau}_{h}, \mathbf{v}_{h}\right), \\
\psi_{h} \int_{\Omega} \operatorname{tr}\left(\boldsymbol{\sigma}_{h}\right) & =0
\end{aligned}\right.
$$

for all $\left(\boldsymbol{\tau}_{h}, \mathbf{v}_{h}, \psi_{h}\right) \in \mathbf{H}_{h} \times V_{h} \times \mathbb{R}$, where $\mathbf{H}_{h}$ is a finite element subspace of $H(\operatorname{div} ; \Omega)$.

In the next theorem we establish the equivalence between (13) and (21).

Theorem 5 Let $\left(\boldsymbol{\sigma}_{h}, \mathbf{u}_{h}\right) \in \mathbf{H}_{0, h} \times V_{h}$ be the solution of (13). Then, $\left(\boldsymbol{\sigma}_{h}, \mathbf{u}_{h}, 0\right)$ is a solution of (21). Conversely, let $\left(\boldsymbol{\sigma}_{h}, \mathbf{u}_{h}, \varphi_{h}\right) \in \mathbf{H}_{h} \times V_{h} \times \mathbb{R}$ be a solution of (21). Then $\varphi_{h}=0$ and $\left(\boldsymbol{\sigma}_{h}, \mathbf{u}_{h}\right)$ is the solution of (13). In particular, problem (21) has a unique solution.

Proof. Let $\left(\boldsymbol{\sigma}_{h}, \mathbf{u}_{h}\right) \in \mathbf{H}_{0, h} \times V_{h}$ be the solution of (13) and let $\left(\boldsymbol{\tau}_{h}, \mathbf{v}_{h}\right) \in \mathbf{H}_{h} \times V_{h}$. Then, $\boldsymbol{\tau}_{h}=$ $\boldsymbol{\tau}_{0, h}+c_{h} \mathbf{I}$, where $\boldsymbol{\tau}_{0, h} \in \mathbf{H}_{0, h}$ and $c_{h}=\frac{1}{d|\Omega|} \int_{\Omega} \operatorname{tr}\left(\boldsymbol{\tau}_{h}\right)$. We remark that $\operatorname{div}\left(\boldsymbol{\tau}_{h}\right)=\operatorname{div}\left(\boldsymbol{\tau}_{0, h}\right)$ and $\tau_{h}^{\mathrm{d}}=\boldsymbol{\tau}_{0, h}^{\mathrm{d}}$. Therefore,

$$
A\left(\left(\boldsymbol{\sigma}_{h}, \mathbf{u}_{h}\right),\left(\boldsymbol{\tau}_{h}, \mathbf{v}_{h}\right)\right)=A\left(\left(\boldsymbol{\sigma}_{h}, \mathbf{u}_{h}\right),\left(\boldsymbol{\tau}_{0, h}, \mathbf{v}_{h}\right)\right)=F\left(\boldsymbol{\tau}_{0, h}, \mathbf{v}_{h}\right)=F\left(\boldsymbol{\tau}_{h}, \mathbf{v}_{h}\right)
$$

where in the second identity we used that $\left(\boldsymbol{\sigma}_{h}, \mathbf{u}_{h}\right)$ is the solution of $(13)$. We deduce that $\left(\boldsymbol{\sigma}_{h}, \mathbf{u}_{h}, 0\right)$ is a solution of problem $(21)$.

On the other hand, let $\left(\boldsymbol{\sigma}_{h}, \mathbf{u}_{h}, \varphi_{h}\right) \in \mathbf{H}_{h} \times V_{h} \times \mathbb{R}$ be a solution of (21). Then, taking $\left(\boldsymbol{\tau}_{h}, \mathbf{v}_{h}\right)=$ $(\mathbf{I}, \mathbf{0})$ in the first equation of $(21)$, it is easy to see that $\varphi_{h}=0$. Since the second equation of $(21)$ implies that $\boldsymbol{\sigma}_{h} \in \mathbf{H}_{0, h}$, we have that $\left(\boldsymbol{\sigma}_{h}, \mathbf{u}_{h}\right)$ is the solution of problem (13).

The numerical experiments were performed with the finite element toolbox ALBERTA using refinement by recursive bisection [27]. The solution of the corresponding linear system is computed using the backslash operator of MATLAB. Given the features of system (21), MATLAB chooses an LU solver (UMFPACK routines) to compute the solution. We present numerical experiments for the finite element pairs $\left(\mathbf{H}_{0, h}, V_{h},\right)$ given by $\left(\left[\mathcal{R} \mathcal{T}_{0}^{\mathrm{t}}\right]^{2},\left[\mathcal{L}_{1}\right]^{2}\right),\left(\left[\mathcal{R} \mathcal{T}_{1}^{\mathrm{t}}\right]^{2},\left[\mathcal{L}_{2}\right]^{2}\right)$ and $\left(\left[\mathcal{B D} \mathcal{M}_{1}^{\mathrm{t}}\right]^{2},\left[\mathcal{L}_{1}\right]^{2}\right)$ and $\left(\left[\mathcal{R} \mathcal{T}_{0}^{\mathrm{t}}\right]^{3},\left[\mathcal{L}_{1}\right]^{3}\right)$

We use the standard adaptive finite element method (AFEM) based on the loop:

$$
\text { SOLVE } \rightarrow \text { ESTIMATE } \rightarrow \text { MARK } \rightarrow \text { REFINE. }
$$

Hereafter, we replace the subscript $h$ by $k$, where $k$ is the counter of the adaptive loop. Then, given a mesh $\mathcal{T}_{k}$, the procedure SOLVE is a direct solver for computing the discrete solution $\left(\boldsymbol{\sigma}_{k}, \mathbf{u}_{k}\right)$. 

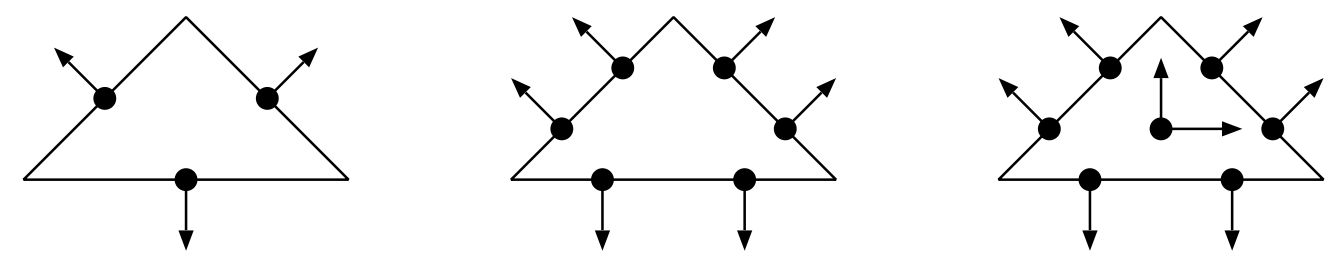

Figure 1: Degrees of freedom for $\mathcal{R} \mathcal{T}_{0}$ (left), $\mathcal{B D} \mathcal{M}_{1}$ (center) and $\mathcal{R} \mathcal{T}_{1}$ (right) on a triangle $T$

ESTIMATE calculates the error indicators $\theta_{k}(T)$ for all $T \in \mathcal{T}_{k}$ depending on the computed solution and the data. Based on the values of $\left\{\theta_{k}(T)\right\}_{T \in \mathcal{T}_{k}}$, the procedure MARK generates a set of marked elements subject to refinement. For the elements selection, we rely on the maximum strategy: Given a threshold $\gamma \in(0,1]$, any element $T^{\prime} \in \mathcal{T}_{k}$ with

$$
\theta_{k}\left(T^{\prime}\right)>\gamma \max _{T \in \mathcal{T}_{k}} \theta_{k}(T)
$$

is marked for refinement. Finally, the procedure REFINE creates a conforming refinement $\mathcal{T}_{k+1}$ of $\mathcal{T}_{k}$, bisecting $d$ times $(d=2$ or 3$)$ all marked elements.

We will compare the performance of a finite element method based on uniform refinement (U) with the adaptive method (A) that we have described above.

In order to get the best ellipticity constant $C_{\text {ell }}$ in Lemma 2 and taking into account that $c_{1} \in(0,1)$ is unknown, we choose $\kappa_{1}$ and $\kappa_{2}$ to be:

$$
\kappa_{1}=\frac{\nu}{1+\nu^{2}+d\|\mathbf{a}\|_{\left[L^{\infty}(\Omega)\right]^{d}}^{2}}, \quad \kappa_{2}=\frac{\nu\left(1+d\|\mathbf{a}\|_{\left[L^{\infty}(\Omega)\right]^{d}}^{2}\right)}{1+\nu^{2}+d\|\mathbf{a}\|_{\left[L^{\infty}(\Omega)\right]^{d}}^{2}},
$$

that satisfy the hypotheses of Lemma 2 .

In what follows, DOFs stands for the total number of degrees of freedom (unknowns) of (21) and we define the individual errors

$$
e_{k}(\boldsymbol{\sigma}):=\left\|\boldsymbol{\sigma}-\boldsymbol{\sigma}_{k}\right\|_{H(\operatorname{div} ; \Omega)}, \quad e_{k}(\mathbf{u}):=\left|\mathbf{u}-\mathbf{u}_{k}\right|_{\left[H^{1}(\Omega)\right]^{d}}, \quad e_{k}(p):=\left\|p-p_{k}\right\|_{L^{2}(\Omega)},
$$

where the pressure $p_{k}$ is recovered as $p_{k}=-\frac{1}{d} \operatorname{tr}\left(\boldsymbol{\sigma}_{k}\right)$, and the total error

$$
e_{k}(\boldsymbol{\sigma}, \mathbf{u}):=\left(e_{k}(\boldsymbol{\sigma})^{2}+e_{k}(\mathbf{u})^{2}\right)^{1 / 2}
$$

The efficiency index with respect to the error estimator $\theta_{k}$ is defined as eff ${ }_{k}:=e_{k}(\boldsymbol{\sigma}, \mathbf{u}) / \theta_{k}$.

We present four examples. The aim of the first one is to confirm Theorem 3 , and to verify the efficiency and reliability of the a posteriori error estimator derived in Section 5. We also analyze the influence of relatively small values of the viscosity $\nu$ in the numerical method. The second and third examples are two-dimensional examples in which the solution presents a boundary layer and an inner layer, respectively; they allow us to show the efficiency of the adaptive method. Finally, the fourth example shows the behavior of the algorithms in a three-dimensional problem with a boundary layer; in this case, the role of the a posteriori error estimator is crucial due to the increasing number of DOFs in three-dimensional problems. 

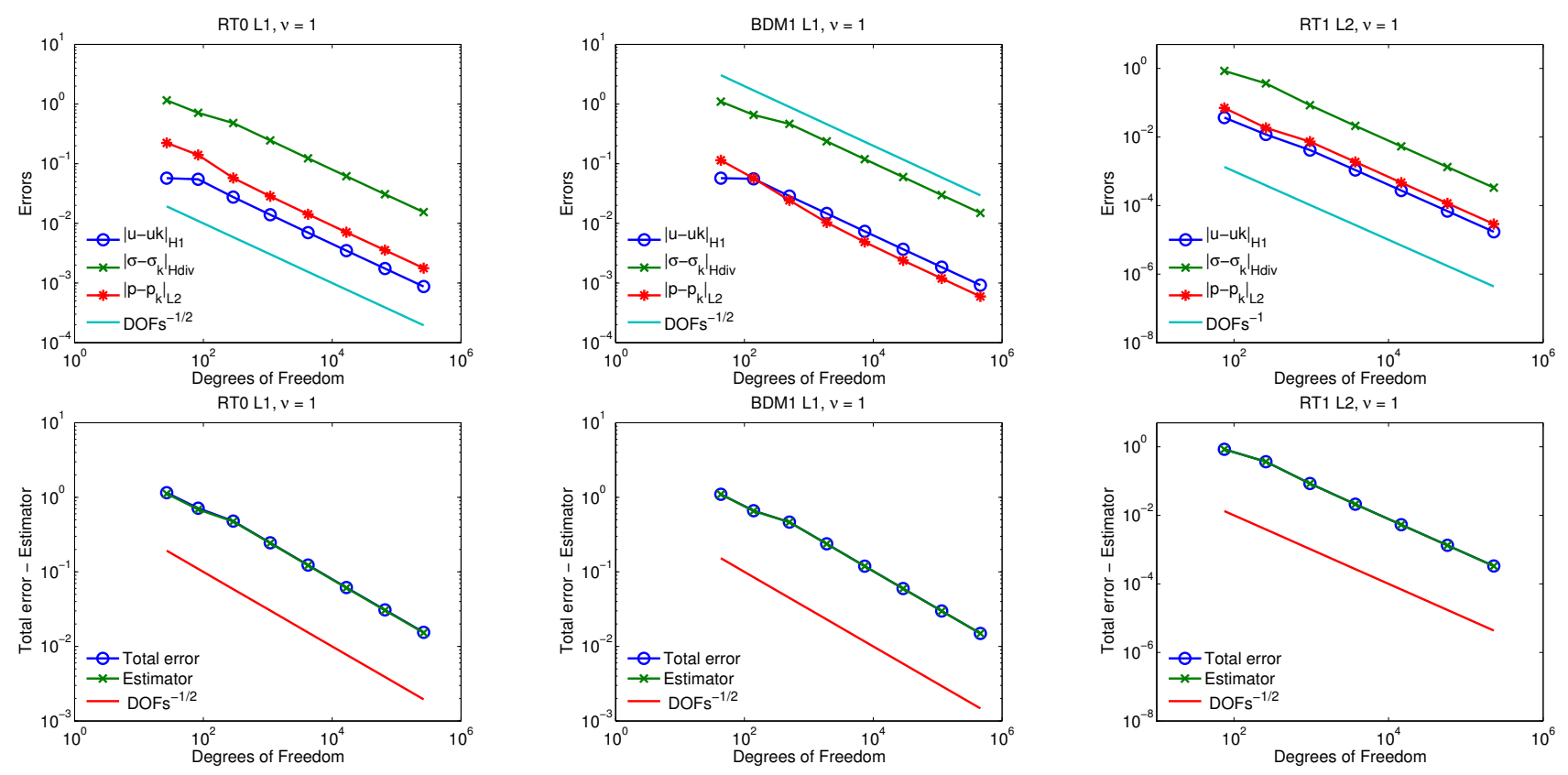

Figure 2: Example 6.1. Decay of errors and estimator for $\left(\left[\mathcal{R} \mathcal{T}_{0}^{\mathrm{t}}\right]^{2},\left[\mathcal{L}_{1}\right]^{2}\right),\left(\left[\mathcal{B D} \mathcal{M}_{1}^{\mathrm{t}}\right]^{2},\left[\mathcal{L}_{1}\right]^{2}\right)$ and $\left(\left[\mathcal{R} \mathcal{T}_{1}^{\mathrm{t}}\right]^{2},\left[\mathcal{L}_{2}\right]^{2}\right)$ and $\nu=1$.

\subsection{A smooth example}

Let $\Omega=(0,1)^{2}, \phi(x, y)=x^{2}(1-x)^{2} y^{2}(1-y)^{2}$ and $\mathbf{a}=(2,3)$. We choose $\mathbf{f}$ so that the exact solution of problem (1) is:

$$
\mathbf{u}=\operatorname{curl} \phi=\left(\frac{\partial \phi}{\partial y},-\frac{\partial \phi}{\partial x}\right), \quad p=\exp \left(-10\left((x-1 / 2)^{2}+(y-1 / 2)^{2}\right)\right)-p_{m},
$$

where $p_{m} \in \mathbb{R}$ is such that $\int_{\Omega} p=0$. We remark that $\mathbf{u}=\mathbf{0}$ on $\Gamma$.

We solve this problem for different values of the viscosity, ranging from 1 to $10^{-6}$, with the finite element pairs $\left(\left[\mathcal{R T}_{0}^{\mathrm{t}}\right]^{2},\left[\mathcal{L}_{1}\right]^{2}\right),\left(\left[\mathcal{B D}_{\mathcal{M}}^{\mathrm{t}}\right]^{2},\left[\mathcal{L}_{1}\right]^{2}\right)$ and $\left(\left[\mathcal{R} \mathcal{T}_{1}^{\mathrm{t}}\right]^{2},\left[\mathcal{L}_{2}\right]^{2}\right)$ on a sequence of uniform meshes (i.e., in each step the elements of the mesh are bisected twice). Figures 2-8 show the decay of the errors $e_{k}(\boldsymbol{\sigma}), e_{k}(\mathbf{u}), \mathbf{e}_{k}(p)$ versus the DOFs and the behavior of the total error and the estimator. Convergence rates predicted by the theory for the total error (Theorem 3) are attained in all cases (we recall that $h^{k} \sim \mathrm{DOFs}^{-k / d}$ on uniform meshes), independently of the value of the viscosity. This fact shows that the original problem is stabilized correctly. Nevertheless, for the finite element pair $\left(\left[\mathcal{R} \mathcal{T}_{1}^{\mathrm{t}}\right]^{2},\left[\mathcal{L}_{2}\right]^{2}\right)$ and values for the viscosity less than $10^{-3}$, we observe certain instabilities in the first steps of the refinement procedure, although in the asymptotic regime the order is correct.

The errors $e_{k}(\boldsymbol{\sigma})$ and $e_{k}(\mathbf{u})$ decay with the same velocity as the total error. The same happens for the error $\mathbf{e}_{k}(p)$ when $\mathcal{R} \mathcal{T}$-elements are employed. The error $\mathbf{e}_{k}(p)$ presents an apparent superconvergence when $\mathcal{B D} \mathcal{M}$-elements are used and $\nu<10^{-2}$. We are not able to determine if this is because 

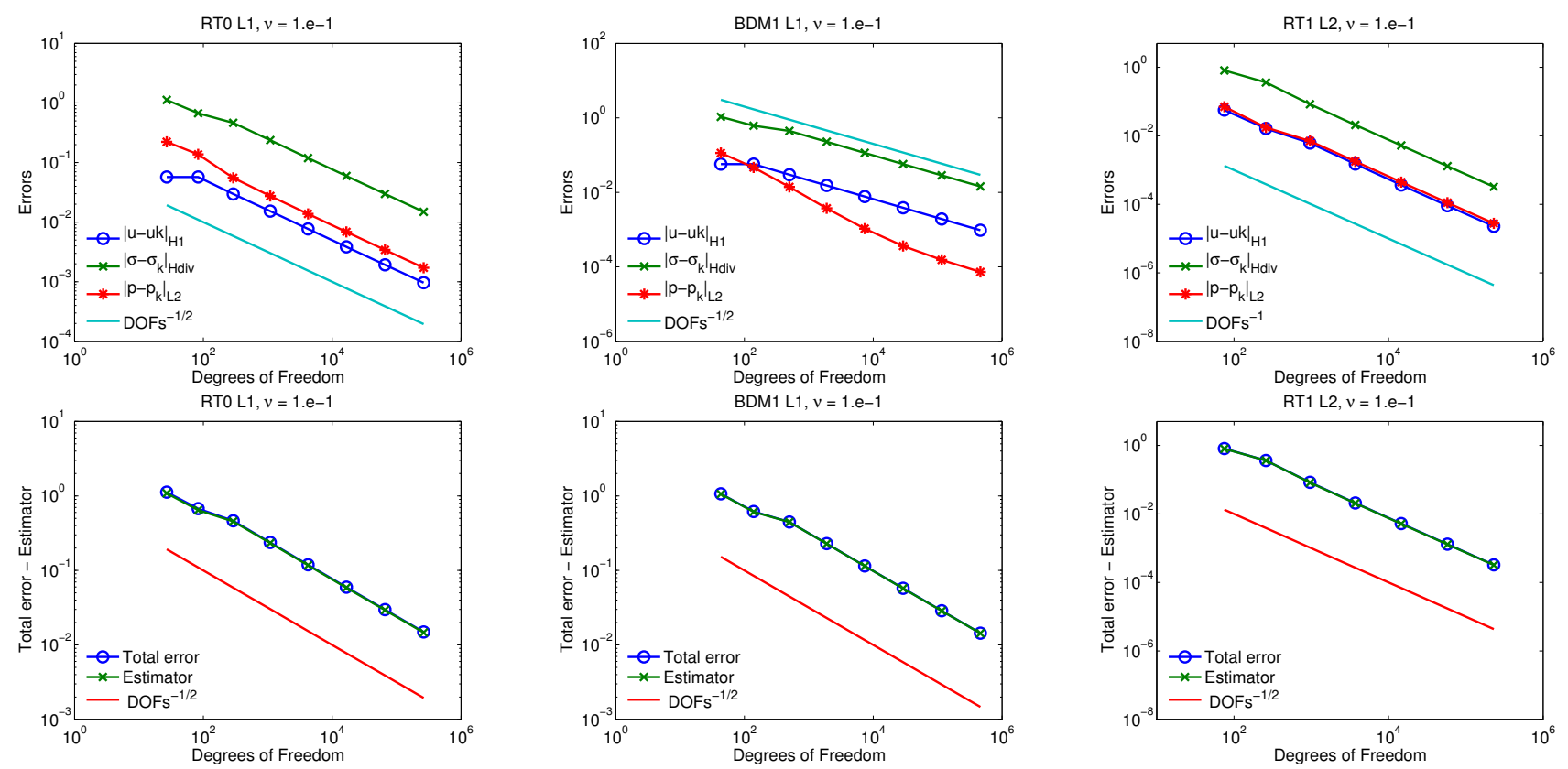

Figure 3: Example 6.1. Decay of errors and estimator for $\left(\left[\mathcal{R} \mathcal{T}_{0}^{\mathrm{t}}\right]^{2},\left[\mathcal{L}_{1}\right]^{2}\right),\left(\left[\mathcal{B D} \mathcal{M}_{1}^{\mathrm{t}}\right]^{2},\left[\mathcal{L}_{1}\right]^{2}\right)$ and $\left(\left[\mathcal{R} \mathcal{T}_{1}^{\mathrm{t}}\right]^{2},\left[\mathcal{L}_{2}\right]^{2}\right)$ and $\nu=10^{-1}$.
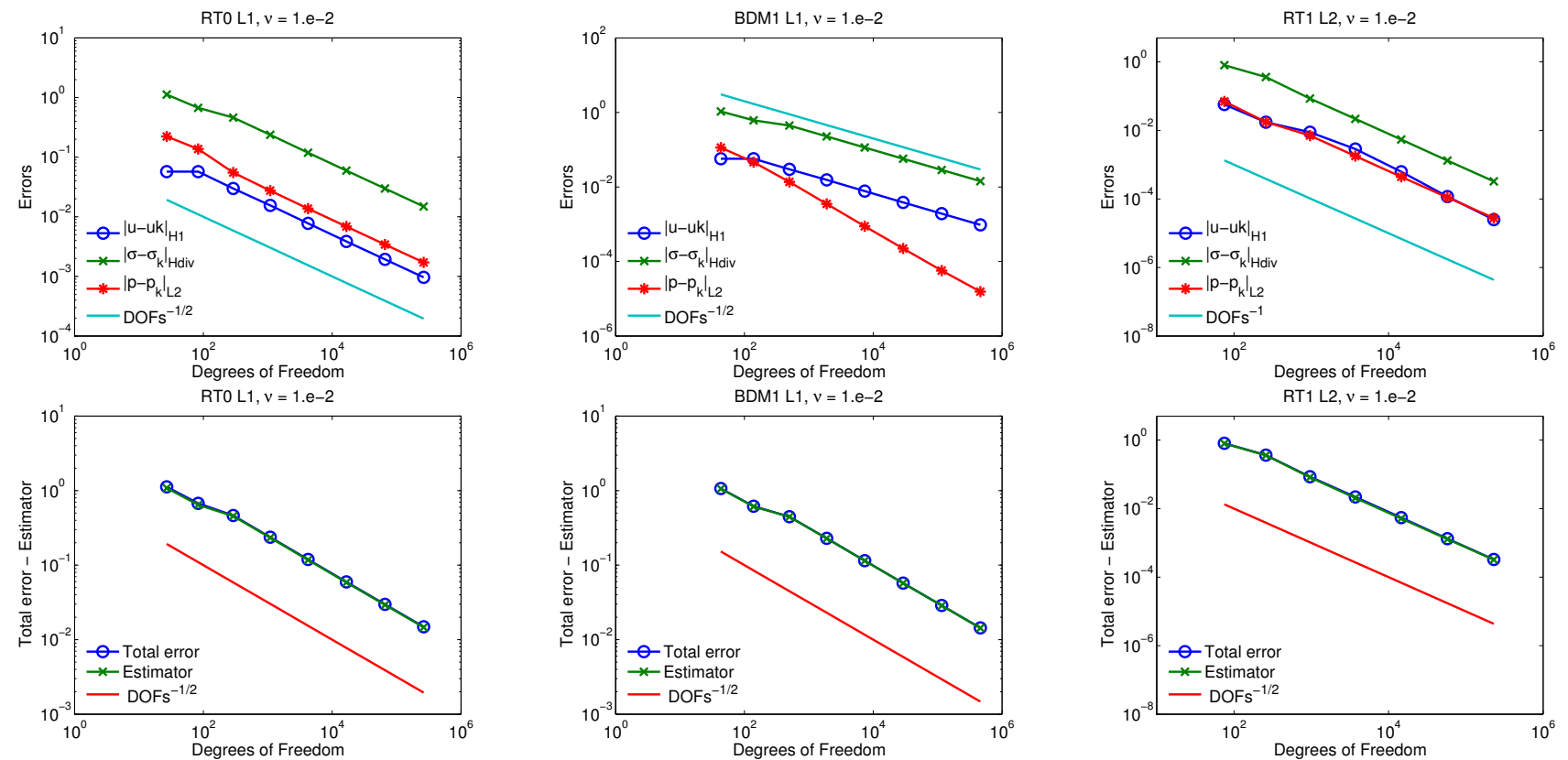

Figure 4: Example 6.1. Decay of errors and estimator for $\left(\left[\mathcal{R} \mathcal{T}_{0}^{\mathrm{t}}\right]^{2},\left[\mathcal{L}_{1}\right]^{2}\right),\left(\left[\mathcal{B D} \mathcal{M}_{1}^{\mathrm{t}}\right]^{2},\left[\mathcal{L}_{1}\right]^{2}\right)$ and $\left(\left[\mathcal{R T}_{1}^{\mathrm{t}}\right]^{2},\left[\mathcal{L}_{2}\right]^{2}\right)$ and $\nu=10^{-2}$. 

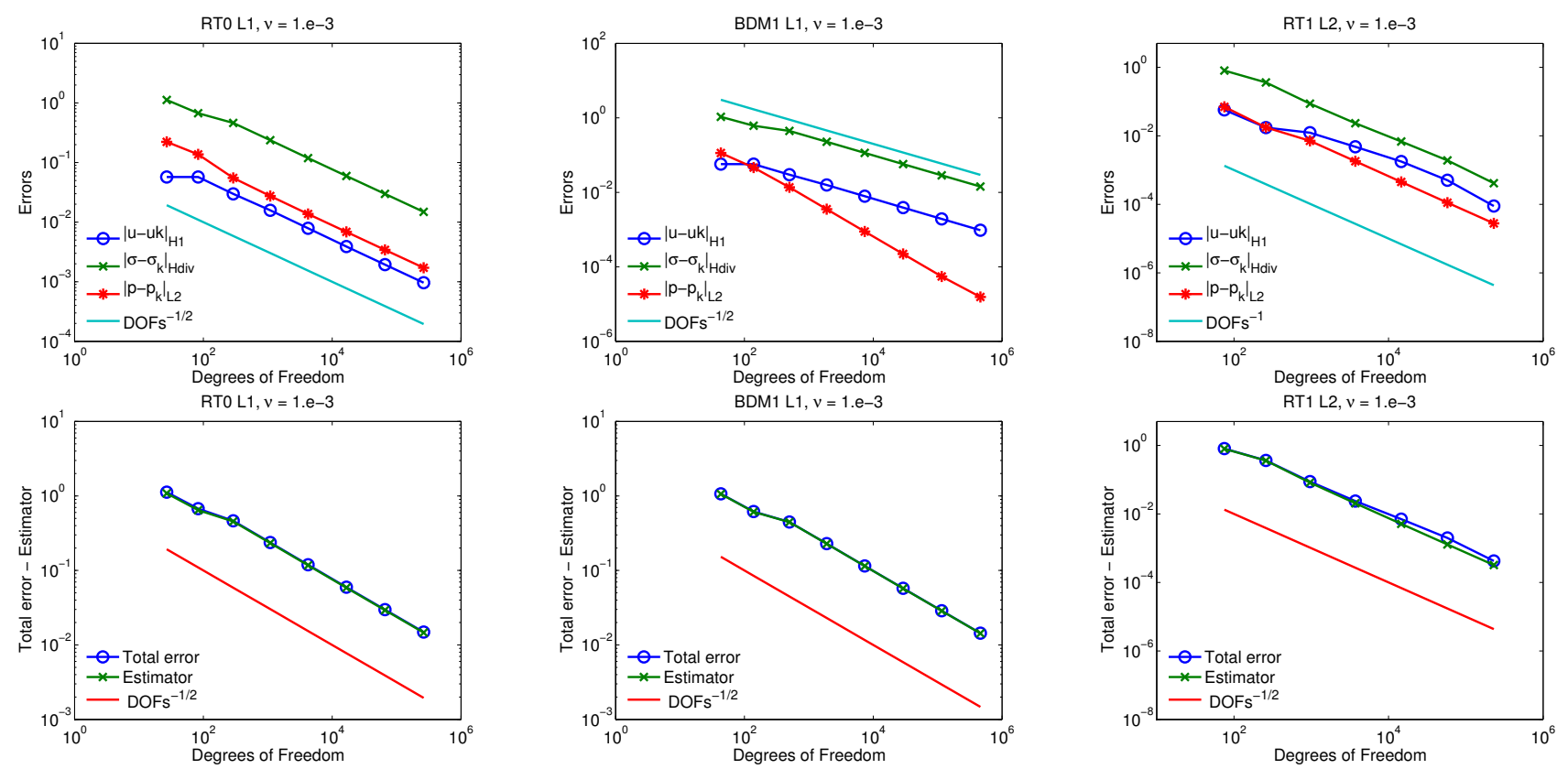

Figure 5: Example 6.1. Decay of errors and estimator for $\left(\left[\mathcal{R} \mathcal{T}_{0}^{\mathrm{t}}\right]^{2},\left[\mathcal{L}_{1}\right]^{2}\right),\left(\left[\mathcal{B D} \mathcal{M}_{1}^{\mathrm{t}}\right]^{2},\left[\mathcal{L}_{1}\right]^{2}\right)$ and $\left(\left[\mathcal{R} \mathcal{T}_{1}^{\mathrm{t}}\right]^{2},\left[\mathcal{L}_{2}\right]^{2}\right)$ and $\nu=10^{-3}$.
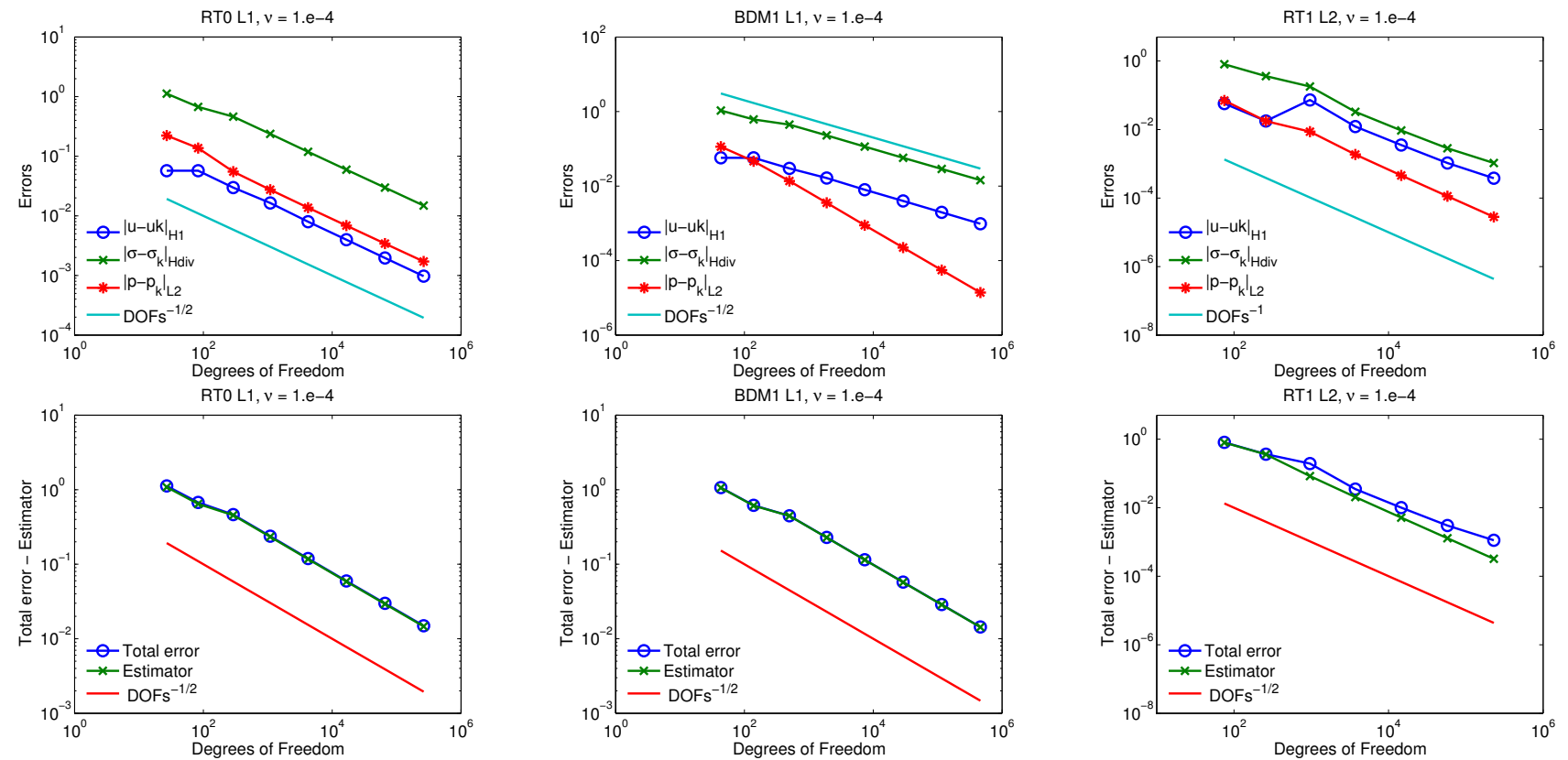

Figure 6: Example 6.1. Decay of errors and estimator for $\left(\left[\mathcal{R} \mathcal{T}_{0}^{\mathrm{t}}\right]^{2},\left[\mathcal{L}_{1}\right]^{2}\right),\left(\left[\mathcal{B D} \mathcal{M}_{1}^{\mathrm{t}}\right]^{2},\left[\mathcal{L}_{1}\right]^{2}\right)$ and $\left(\left[\mathcal{R T}_{1}^{\mathrm{t}}\right]^{2},\left[\mathcal{L}_{2}\right]^{2}\right)$ and $\nu=10^{-4}$. 

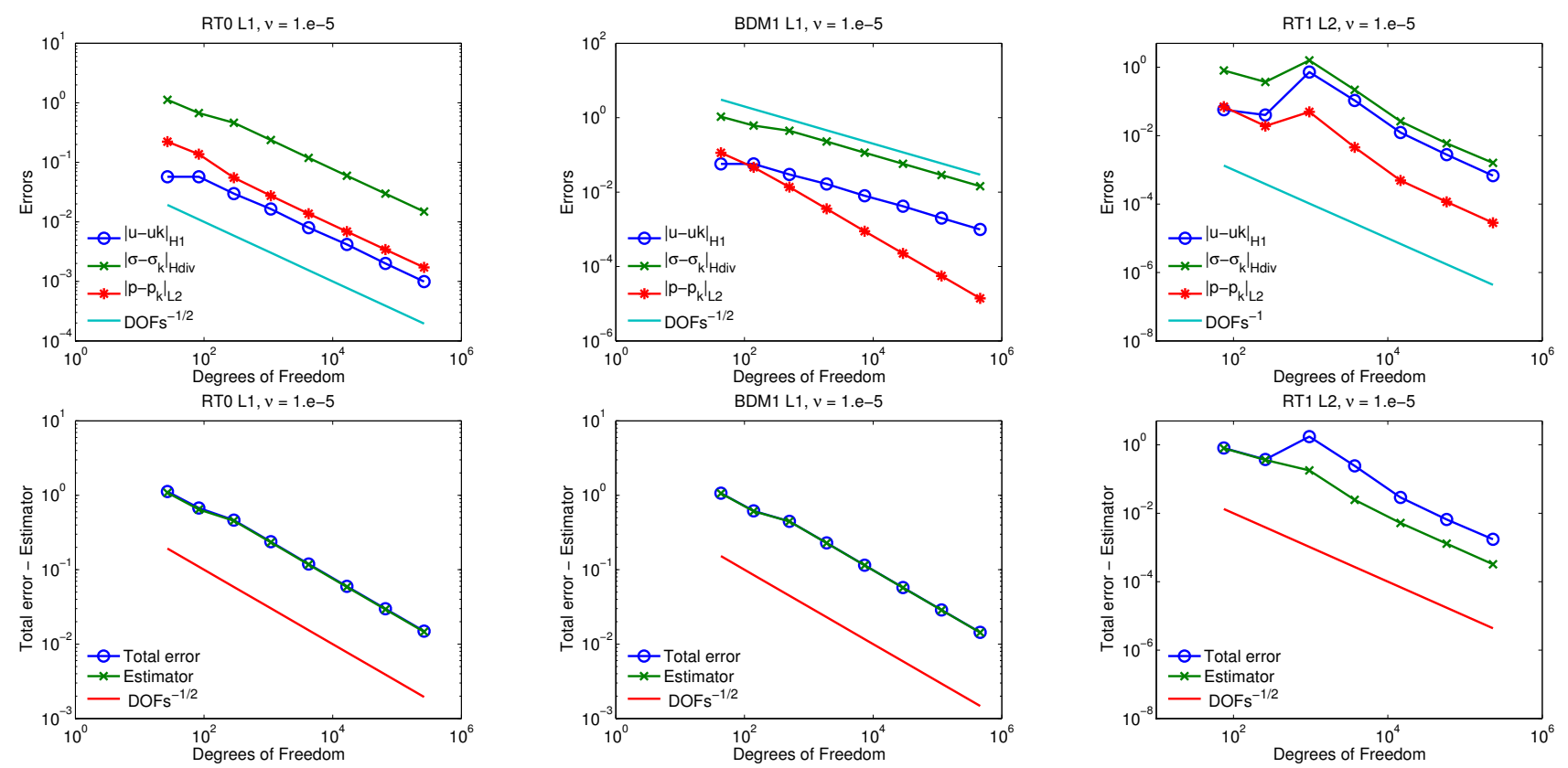

Figure 7: Example 6.1. Decay of errors and estimator for $\left(\left[\mathcal{R} \mathcal{T}_{0}^{\mathrm{t}}\right]^{2},\left[\mathcal{L}_{1}\right]^{2}\right),\left(\left[\mathcal{B D} \mathcal{M}_{1}^{\mathrm{t}}\right]^{2},\left[\mathcal{L}_{1}\right]^{2}\right)$ and $\left(\left[\mathcal{R} \mathcal{T}_{1}^{\mathrm{t}}\right]^{2},\left[\mathcal{L}_{2}\right]^{2}\right)$ and $\nu=10^{-5}$.
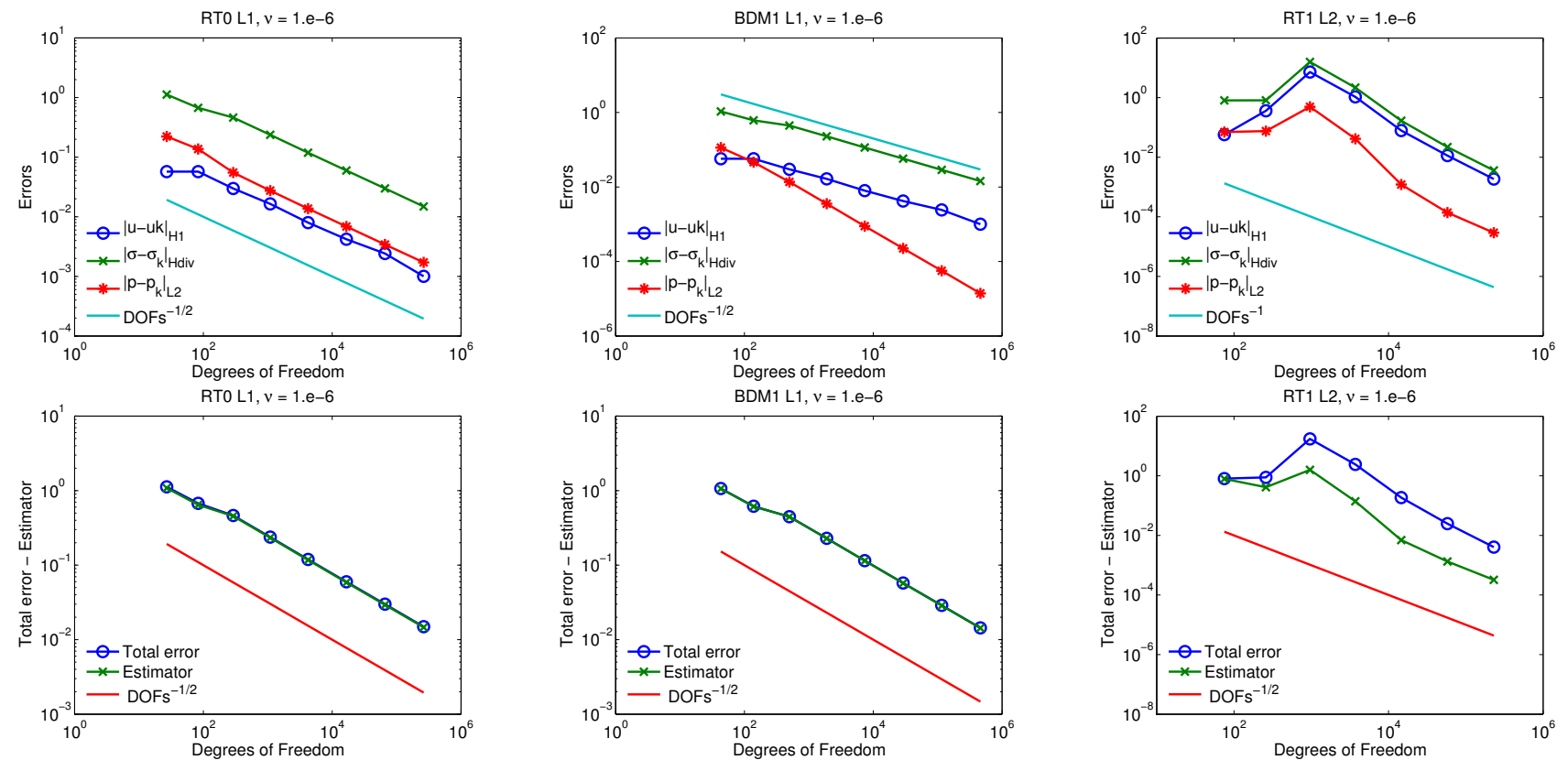

Figure 8: Example 6.1. Decay of errors and estimator for $\left(\left[\mathcal{R} \mathcal{T}_{0}^{\mathrm{t}}\right]^{2},\left[\mathcal{L}_{1}\right]^{2}\right),\left(\left[\mathcal{B D} \mathcal{M}_{1}^{\mathrm{t}}\right]^{2},\left[\mathcal{L}_{1}\right]^{2}\right)$ and $\left(\left[\mathcal{R T}_{1}^{\mathrm{t}}\right]^{2},\left[\mathcal{L}_{2}\right]^{2}\right)$ and $\nu=10^{-6}$. 

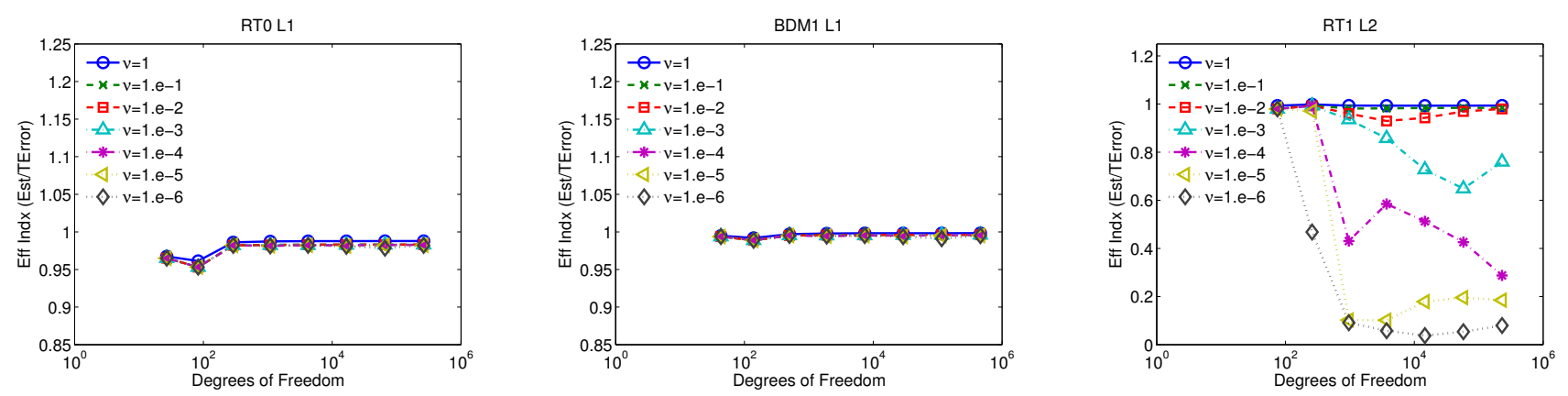

Figure 9: Example 6.1. Efficiency indices for $\left(\left[\mathcal{R} \mathcal{T}_{0}^{\mathrm{t}}\right]^{2},\left[\mathcal{L}_{1}\right]^{2}\right),\left(\left[\mathcal{B D} \mathcal{M}_{1}^{\mathrm{t}}\right]^{2},\left[\mathcal{L}_{1}\right]^{2}\right)$ and $\left(\left[\mathcal{R} \mathcal{T}_{1}^{\mathrm{t}}\right]^{2},\left[\mathcal{L}_{2}\right]^{2}\right)$ and the different values of $\nu$.

the graph of the error in the pressure variable is still in the preasymptotic regime or if for this finite element pair the error $\left\|\boldsymbol{\sigma}-\boldsymbol{\sigma}_{k}\right\|_{L^{2}(\Omega)}$ converges quadratically. In all cases the total error and the estimator have the same decay, which confirms that they are equivalent.

Figure 9 shows the efficiency indices for the different finite element pairs and viscosities considered. Efficiency indices are around 1 and independent of the value of $\nu$ for the families $\left(\left[\mathcal{R} \mathcal{T}_{0}^{\mathrm{t}}\right]^{2},\left[\mathcal{L}_{1}\right]^{2}\right)$ and $\left(\left[\mathcal{B D} \mathcal{M}_{1}^{\mathrm{t}}\right]^{2},\left[\mathcal{L}_{1}\right]^{2}\right)$. For the finite element pair $\left(\left[\mathcal{R} \mathcal{T}_{1}^{\mathrm{t}}\right]^{2},\left[\mathcal{L}_{2}\right]^{2}\right)$, efficiency indices seem to degrade as $\nu$ decreases, according to Theorem 4.

\subsection{Two-dimensional boundary layer}

In this example we consider $\Omega=(0,1)^{2}, \nu=10^{-3}$ and $\mathbf{a}=(1,1)$. We define the potential $\phi(x, y)=$ $x^{2} y^{2}\left(1-e^{\lambda(x-1)}\right)^{2}\left(1-e^{\lambda(y-1)}\right)^{2}$, with $\lambda=0.5 / \sqrt{\nu}$, and choose $\mathbf{f}$ such that the exact solution of problem (1) is:

$$
\mathbf{u}=\operatorname{curl} \phi=\left(\frac{\partial \phi}{\partial y},-\frac{\partial \phi}{\partial x}\right), \quad \quad p=e^{x+y}-p_{m},
$$

where $p_{m} \in \mathbb{R}$ is such that $\int_{\Omega} p=0$. We remark that the velocity $\mathbf{u}$ exhibits a boundary layer around the lines $x=1$ and $y=1$. We also remark that $\mathbf{u}=\mathbf{0}$ on $\Gamma$. In the adaptive procedure we chose $\gamma=0.4$.

Figure 10 shows the decay of the individual errors, $e_{k}(\boldsymbol{\sigma}), e_{k}(\mathbf{u})$ and $e_{k}(p)$ for the adaptive and uniform refinements when the finite element pair $\left(\left[\mathcal{R} \mathcal{T}_{0}^{\mathrm{t}}\right]^{2},\left[\mathcal{L}_{1}\right]^{2}\right)$ is used. We observe a fast convergence of the adaptive procedure for the variables of interest (velocity and pseudostress) as a consequence of the localization of the boundary layer (see Figure 12). We also remark that the error in the pressure variable is several orders of magnitude smaller than the error in the velocity and the pseudostres variables, so that the a posteriori error estimator does not see this error yet.

In Figure 11-left, we can observe the total error and estimator for the uniform and adaptive refinements. From this graph, we conclude that the adaptive algorithm is more competitive than the uniform procedure. Figure 11-right shows the efficiency indices, that tend to stabilize around 0.4.

Figure 13 shows the velocity module for iterations 5, 10 and 15 of the adaptive procedure. In Figures 14 and 15 we can observe the profile of the first component of the velocity near the boundary 

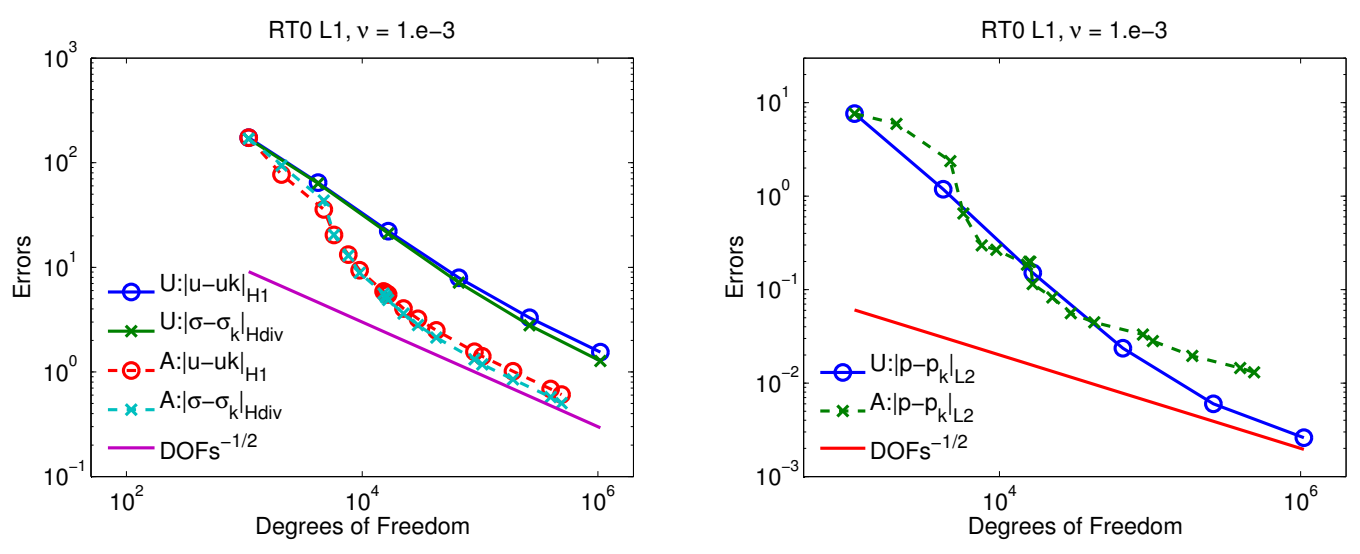

Figure 10: Example 6.2. Errors decay for the adaptive (A) and uniform (U) procedures.
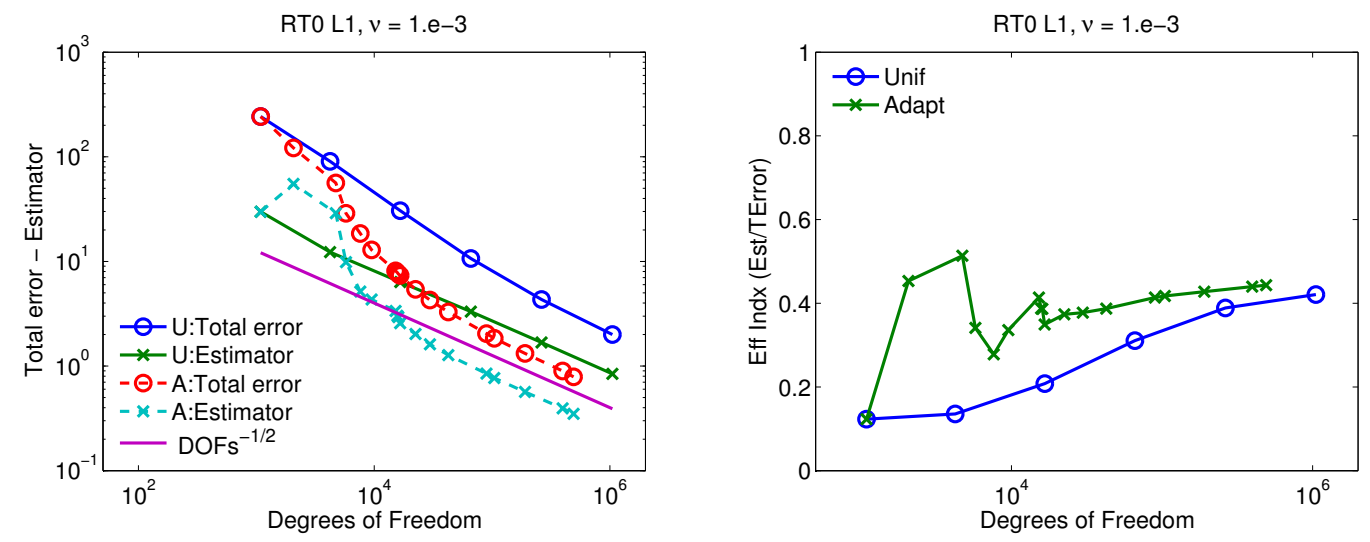

Figure 11: Example 6.2. Decay of total error and estimator (left) and efficiency indices for the adaptive (A) and uniform (U) procedures
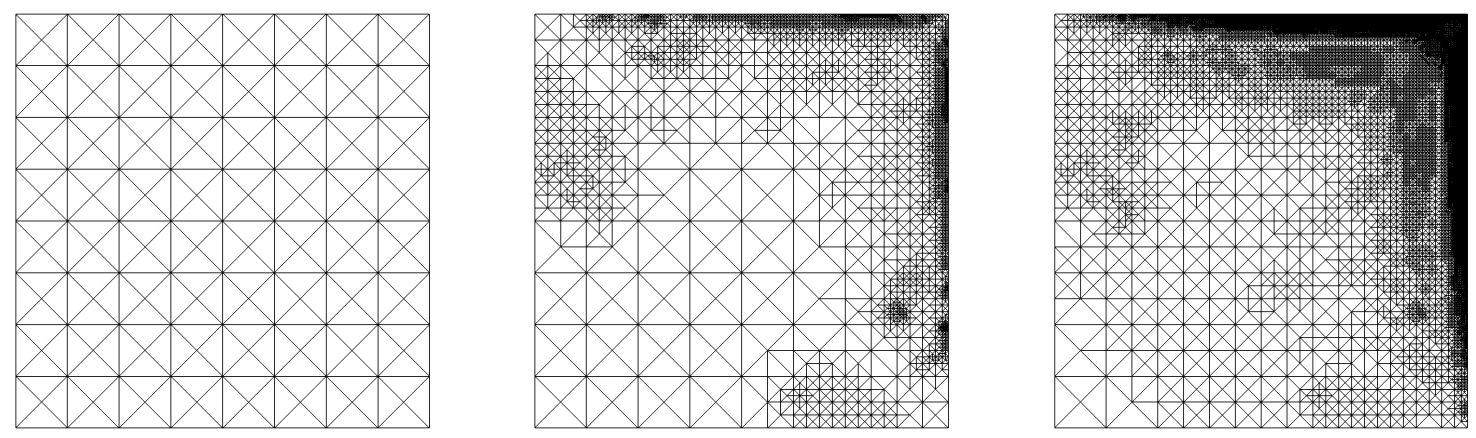

Figure 12: Example 6.2. Meshes generated by the adaptive algorithm in iterations 0, 10 and 15 

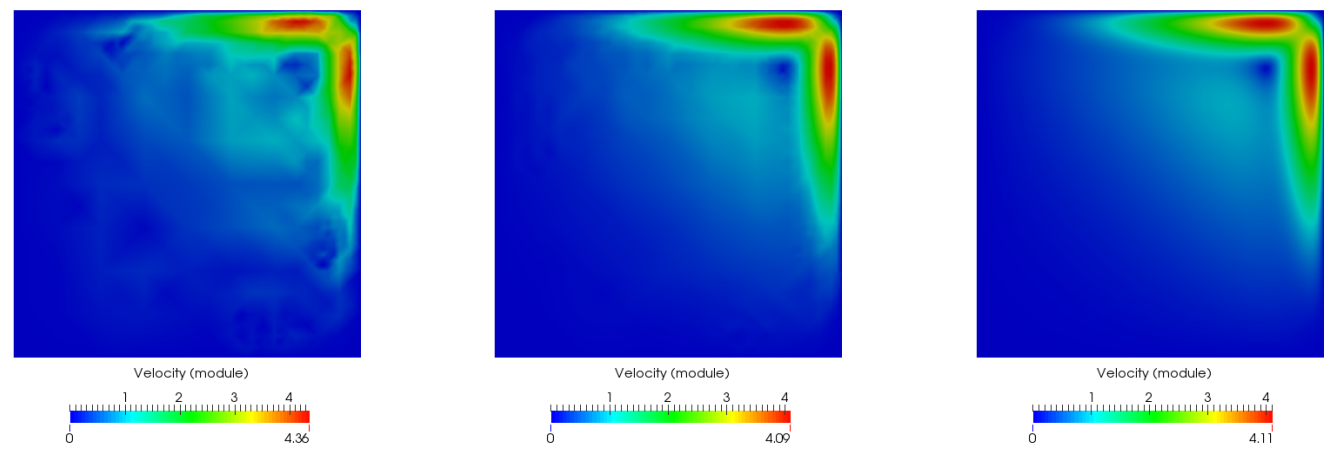

Figure 13: Example 6.2. Velocity module in iterations 5, 10 and 15 of procedure A

layers. We notice that the method captures the boundary layer correctly when the mesh is finer enough in case of uniform refinement or as the adaptive procedure advances, respectively. Finally, Figure 16 shows the pressure obtained in iterations 5, 12 and 17 of the adaptive procedure. We observe some oscillations in the first steps of the adaptive algorithm that diminish later as the algorithm advances.

\subsection{Two-dimensional inner layer}

Let $\Omega=(0,1)^{2}, \nu=10^{-4}$ and $\mathbf{a}=(1,0)$. We consider $\phi(x, y)=x^{2}(1-x)^{2} y^{2}(1-y)^{2}(1-\tanh ((1 / 2-$ $x) \lambda)$ ) with $\lambda=150$, and choose $\mathbf{f}$ so that the exact solution of problem (1) is:

$$
\mathbf{u}=\operatorname{curl} \phi=\left(\frac{\partial \phi}{\partial y},-\frac{\partial \phi}{\partial x}\right), \quad p=e^{-(x-1 / 2)^{2}}-p_{m},
$$

where $p_{m} \in \mathbb{R}$ is such that $\int_{\Omega} p=0$. We remark that the solution presents an inner layer around the line $x=1 / 2$ and that $\mathbf{u}=\mathbf{0}$ on $\Gamma$. In the adaptive procedure we used $\gamma=0.4$.

Figures 17 and 18 show the decay of the errors and estimator with the DOFs for the uniform and adaptive refinements when the finite element pair $\left(\left[\mathcal{R} \mathcal{T}_{0}^{\mathrm{t}}\right]^{2},\left[\mathcal{L}_{1}\right]^{2}\right)$ is used. The same observations of the previous example apply. Again, the adaptive procedure obtains quasi-optimal meshes and it is more competitive than the uniform procedure concerning the reduction of the total error. We have to take into account that the adaptive algorithm is leaded by an a posteriori error estimator that is equivalent to the total error. Then, it can happen, as can be observed from Figure 17, that the errors in the pressure variable can be smaller in the uniform sequence than in the adaptive sequence. Since in this case the pressure is a smooth function, the refinement that captures the inner layer of the velocity is not appropriate for a correct approximation of the pressure.

Figure 19 shows the initial mesh and the meshes generated by the adaptive algorithm in iterations 5 and 9 . We remark that the adaptive algorithm detects the inner layer of the velocity. In Figure 20 we present some graphs of the velocity module in iterations 4, 7 and 11. In Figures 21 and 22 we show the velocity profiles near the inner layer. We observe that the oscillations in the velocity profiles that are present in the first steps tend to dissapear when the mesh is finer enough. Finally, in Figure 23 

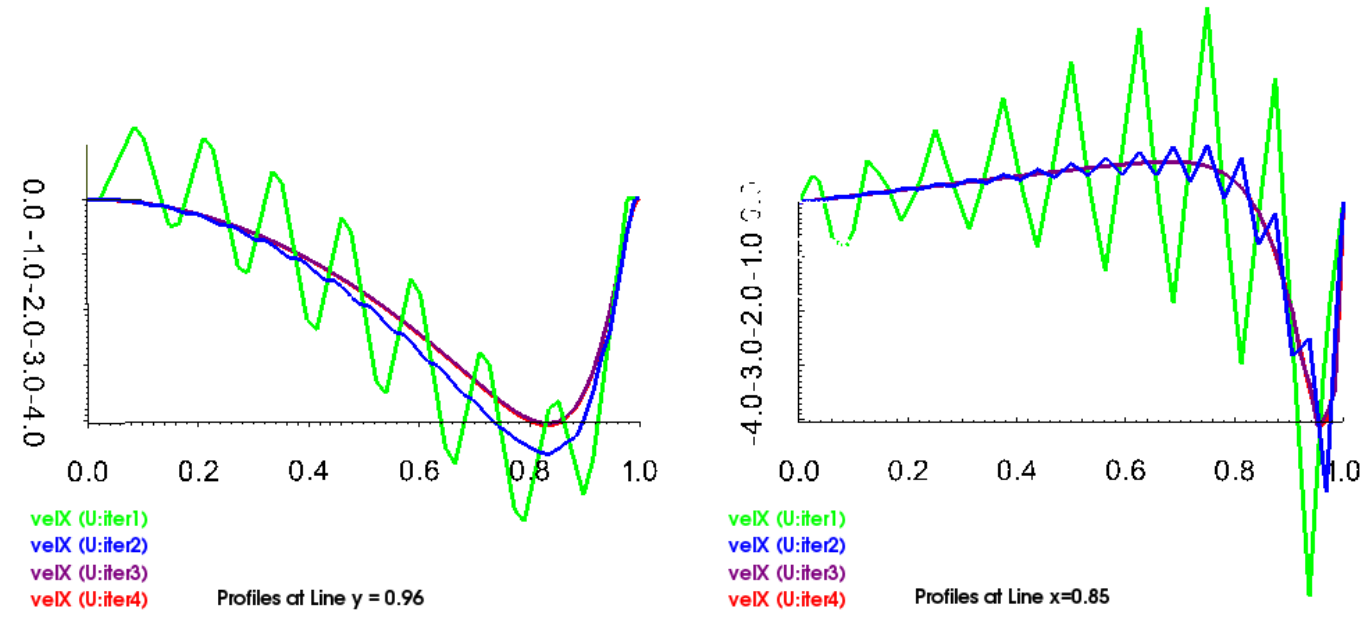

Figure 14: Example 6.2. Velocity profile at $y=0.96$ (left) and $x=0.85$ (right) in different iterations of procedure $\mathrm{U}$
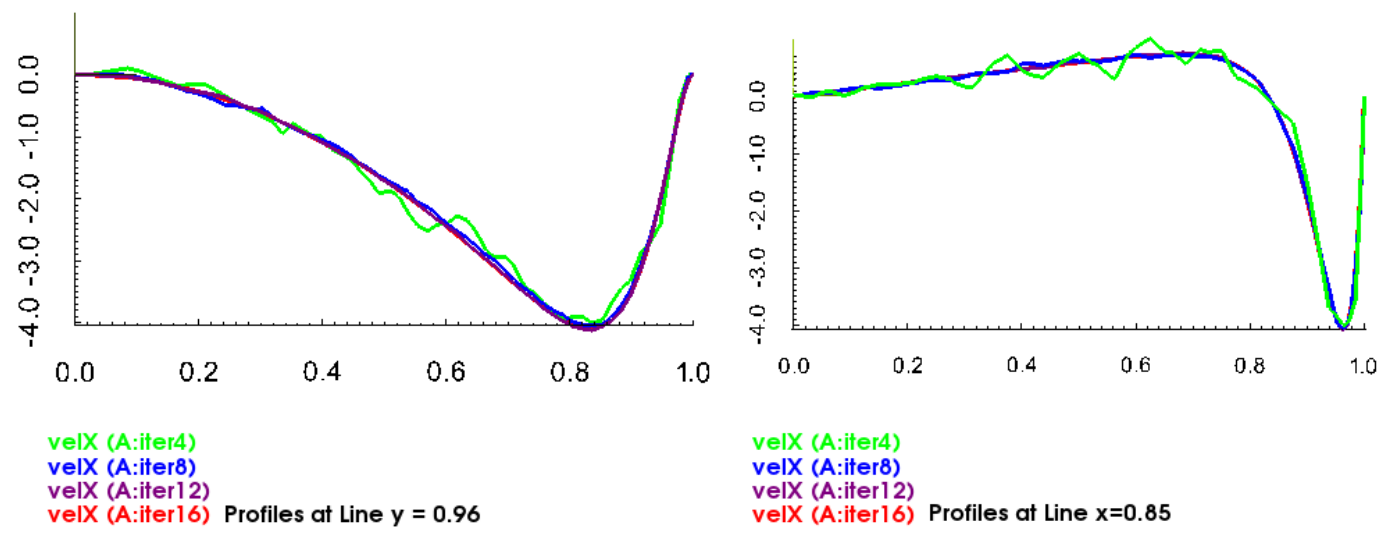

velX (A:iter4)

velX (A:iter8)

velX (A:iter 12)

velX (A:iter16) Profiles at Line $x=0.85$

Figure 15: Example 6.2. Velocity profile at $y=0.96$ (left) and $x=0.85$ (right) in different iterations of procedure A 

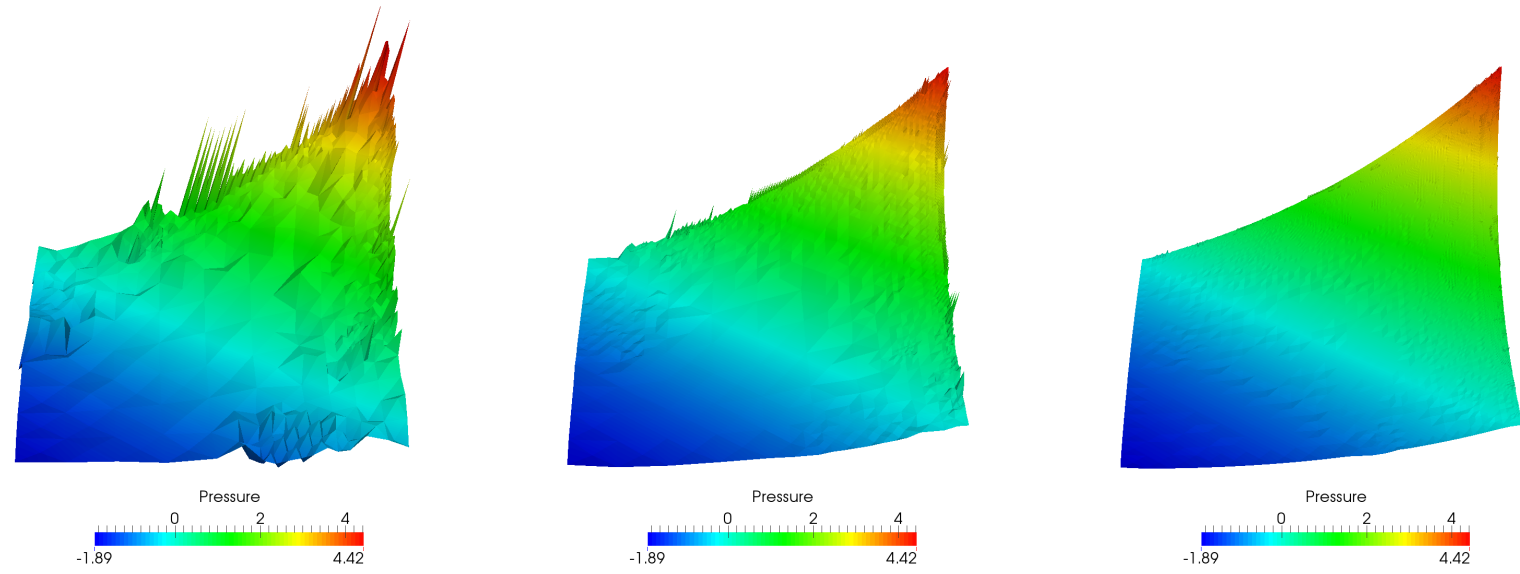

Figure 16: Example 6.2. Pressure in iterations 5, 12 and 17 of procedure A
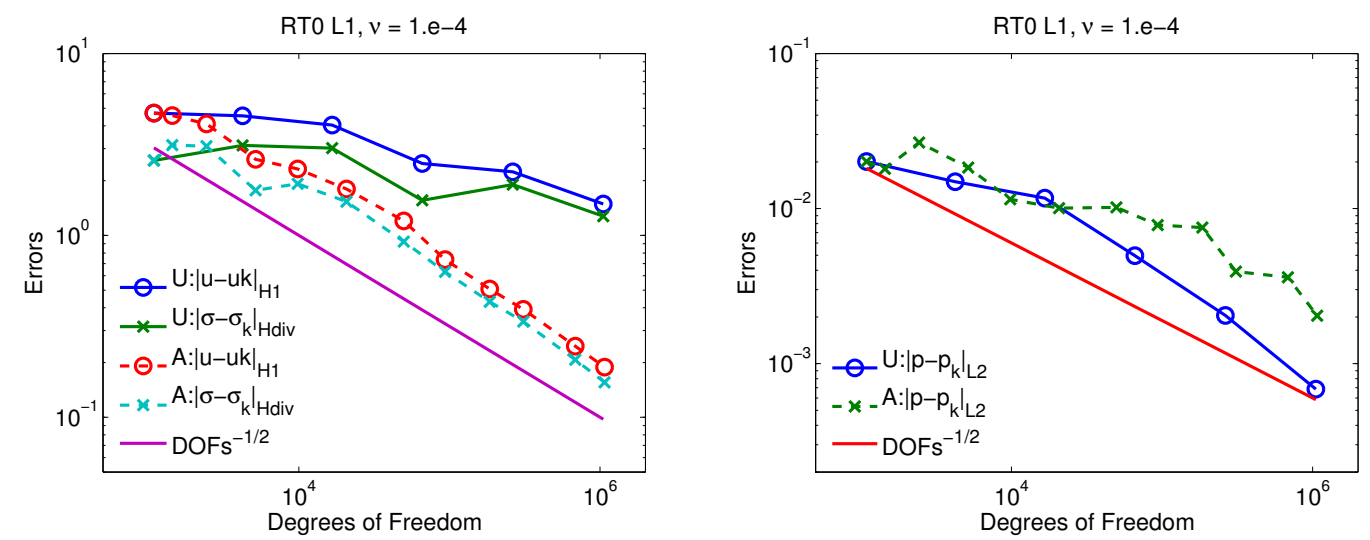

Figure 17: Example 6.3. Errors decay for the adaptive algorithm (A) and the uniform algorithm (U) 

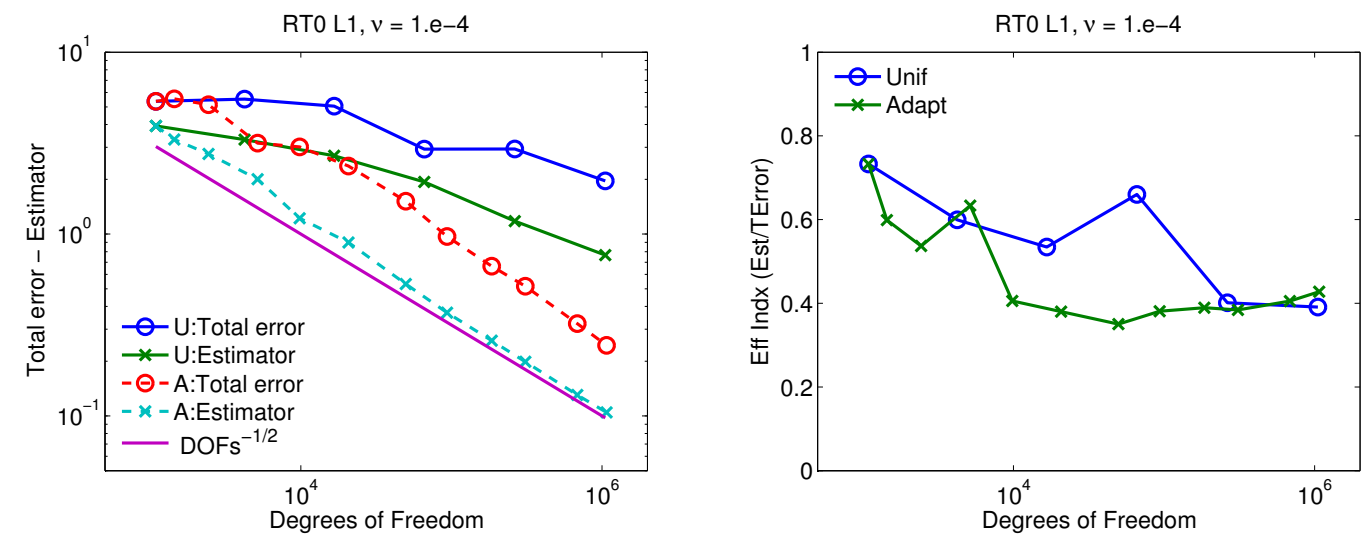

Figure 18: Example 6.3. Decay of the total error and estimator (left) and efficiency indices for the adaptive algorithm (A) and uniform algorithm (U)
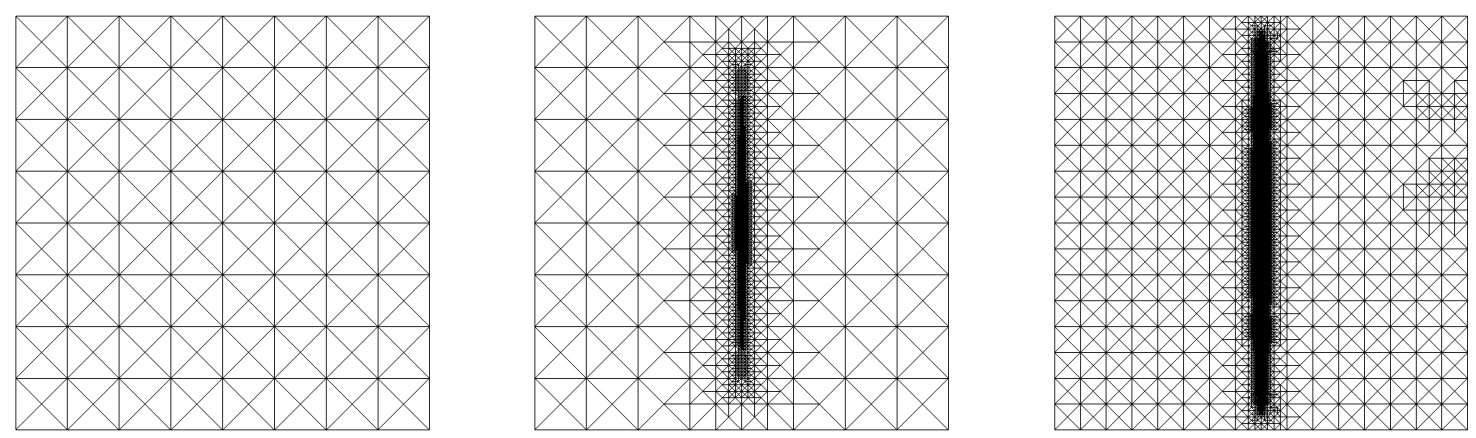

Figure 19: Example 6.3. Meshes generated by the adaptive procedure at iterations 0,5 and 9 .

we present some graphs of the pressure in iterations 4, 8 and 11. Again, in the first iterations there are some instabilities that diminish as the algorithm advances.

\subsection{Three-dimensional boundary layer}

Let $\Omega=(0,1)^{3}, \nu=1$ and $\mathbf{a}=(1,1,0)$. We consider the vector potential

$$
\phi(x, y, z)=x^{2}(1-x)^{2} y^{2}(1-y)^{2} z^{2}\left(1-e^{\lambda(z-1)}\right)^{2}(1,1,1)^{\mathrm{t}}
$$

with $\lambda=20$, and choose $\mathbf{f}$ so that the exact solution of problem (1) is:

$$
\mathbf{u}=\operatorname{curl} \boldsymbol{\phi}=\left(\frac{\partial \phi_{3}}{\partial y}-\frac{\partial \phi_{2}}{\partial z}, \frac{\partial \phi_{1}}{\partial z}-\frac{\partial \phi_{3}}{\partial x}, \frac{\partial \phi_{2}}{\partial x}-\frac{\partial \phi_{1}}{\partial y}\right), \quad p=e^{-10\left((x-1 / 2)^{2}+(y-1 / 2)^{2}+(z-1)^{2}\right)}-p_{m},
$$




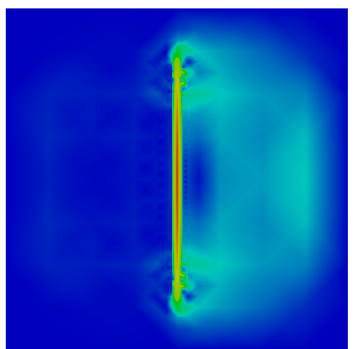

Velocity (module)

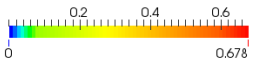

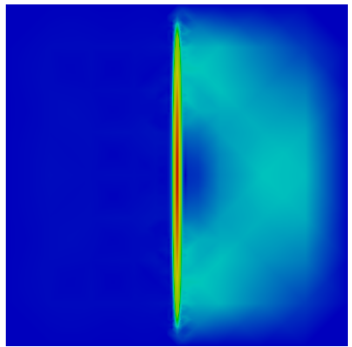

Velocity (module)

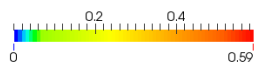

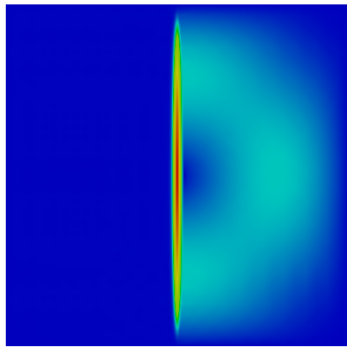

Velocity (module)

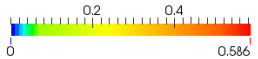

Figure 20: Example 6.3. Module of the velocity field at iterations 4, 7 and 11.
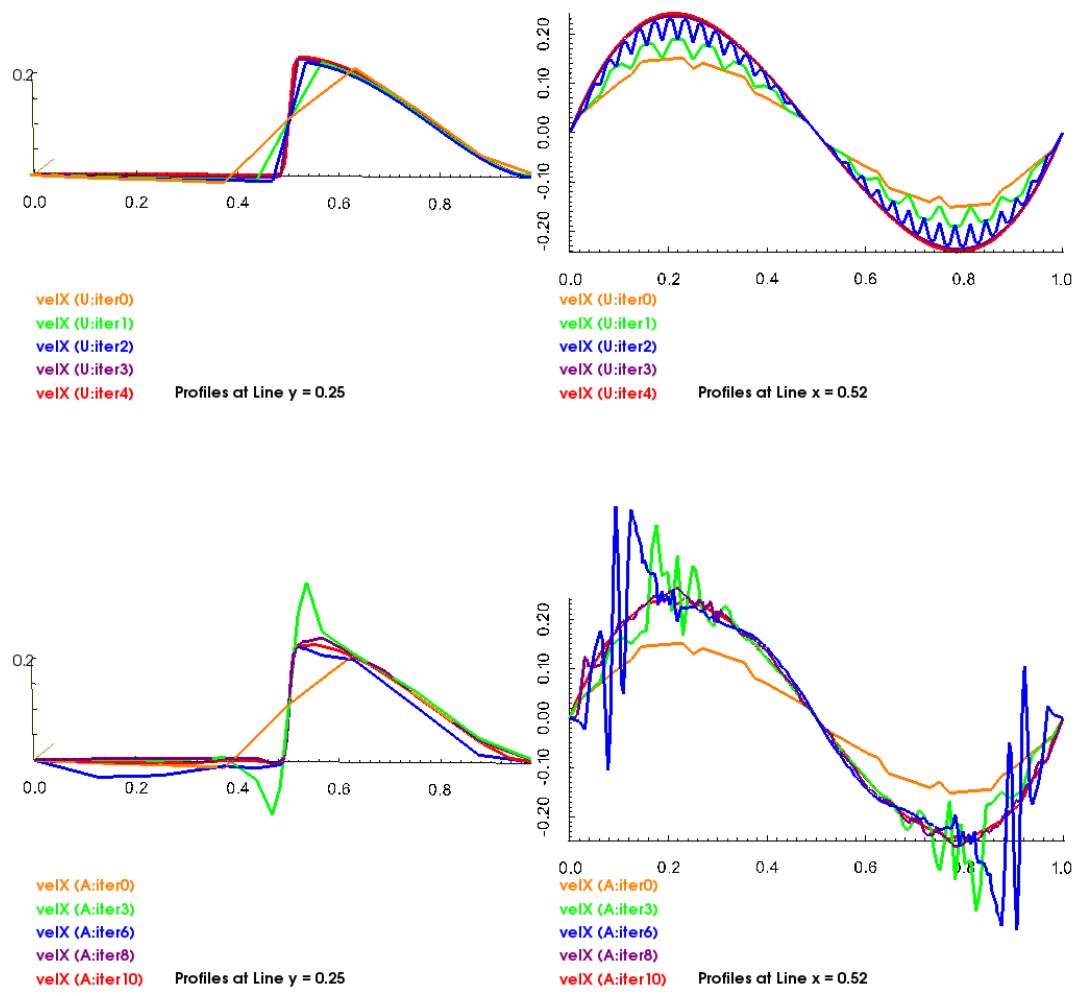

Figure 21: Example 6.3. X-velocity profiles near the boundary layer in different iterations of procedures $\mathrm{U}$ (above) and A (below) 

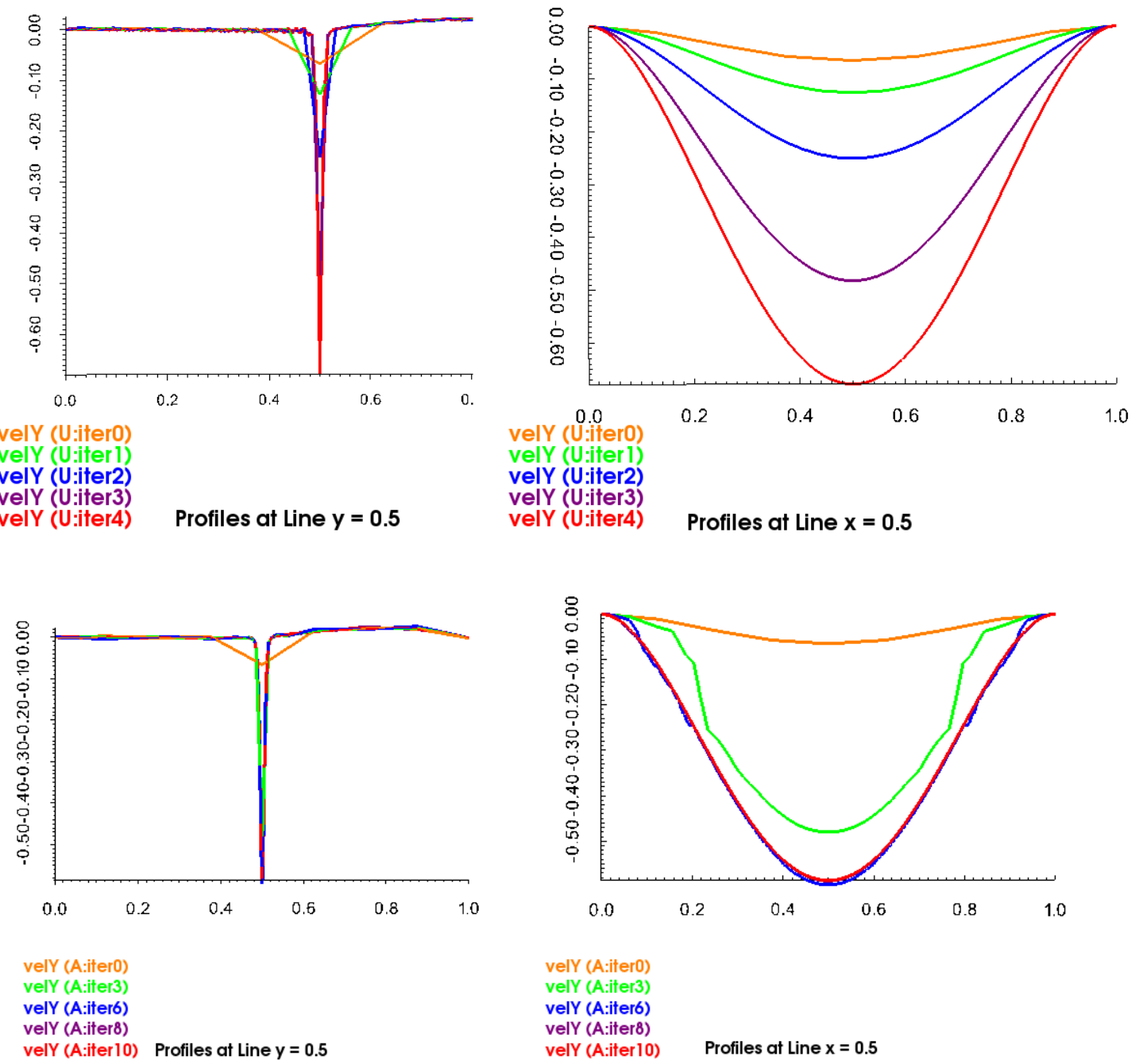

velY (A:iter0)

velY (A:iter3)

velY (A:iter6)

velY (A:iter8)

velY (A:iter 10) Profiles at Line $\mathrm{x}=\mathbf{0 . 5}$

Figure 22: Example 6.3. Y-velocity profiles near the boundary layer in different iterations of procedures $\mathrm{U}$ (above) and A (below) 

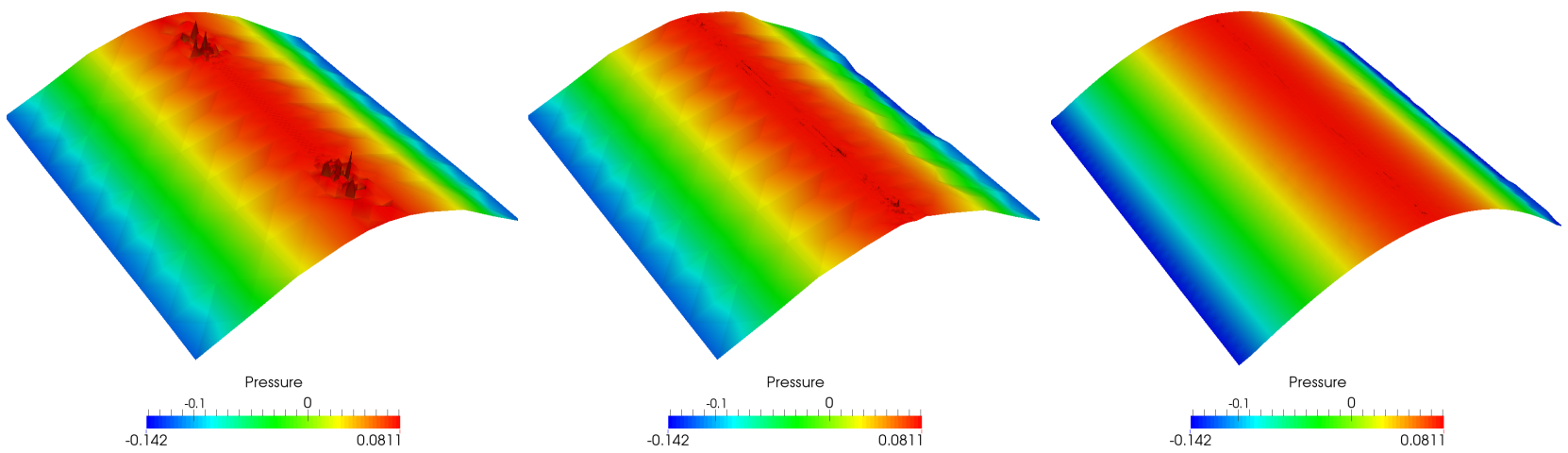

Figure 23: Example 6.3. Pressure at iterations 4, 8 and 11.
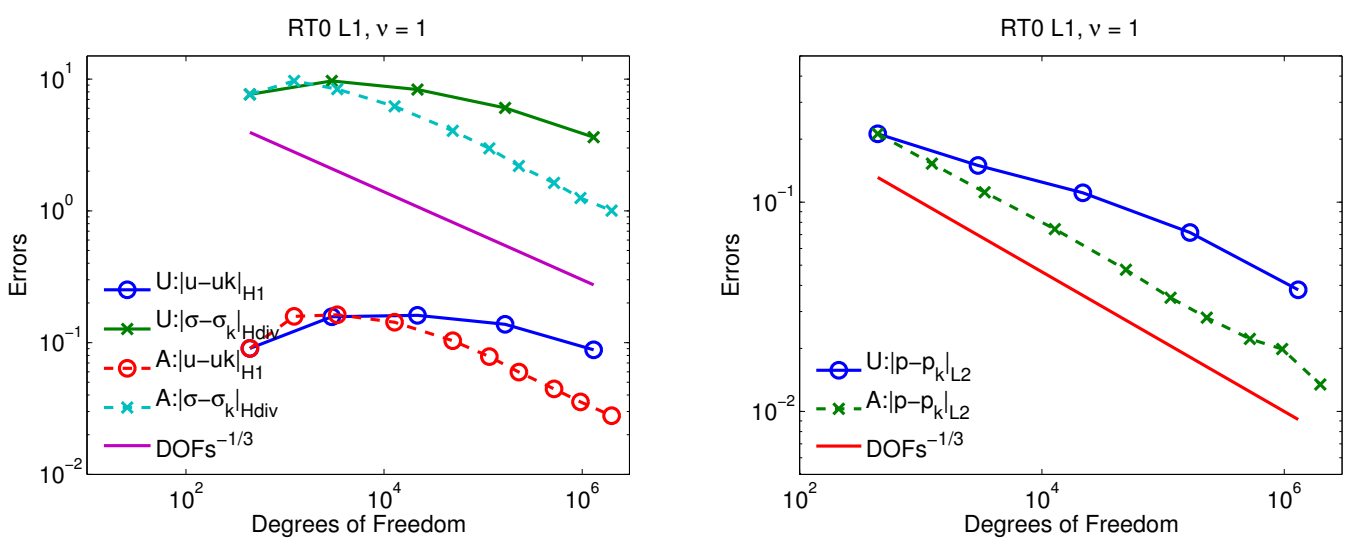

Figure 24: Example 6.4. Errors decay with DOFs for the adaptive (A) and uniform (U) algorithms.

where $p_{m} \in \mathbb{R}$ is such that $\int_{\Omega} p=0$. We remark that the velocity field $\mathbf{u}$ is divergence-free and vanishes on $\Gamma$. In this case, we took $\gamma=0.5$ in the adaptive procedure.

Figures 24 and 25 show the decay of the errors and estimator with the DOFs for the uniform and adaptive refinements when the finite element pair $\left(\left[\mathcal{R} \mathcal{T}_{0}^{\mathrm{t}}\right]^{3},\left[\mathcal{L}_{1}\right]^{3}\right)$ is used. We observe that the adaptive procedure generates quasi-optimal meshes from the third iteration, while the error curves associated to the sequence of uniform meshes exhibit a slow convergence and seem to be at the pre-asymptotic regime. In this example the efficiency index is almost one (see Figure 25-left).

Finally, in Figure 26 we show some meshes generated by the adaptive algorithm. Figures 27 and 28 show the module of the velocity field and the pressure, respectively. 

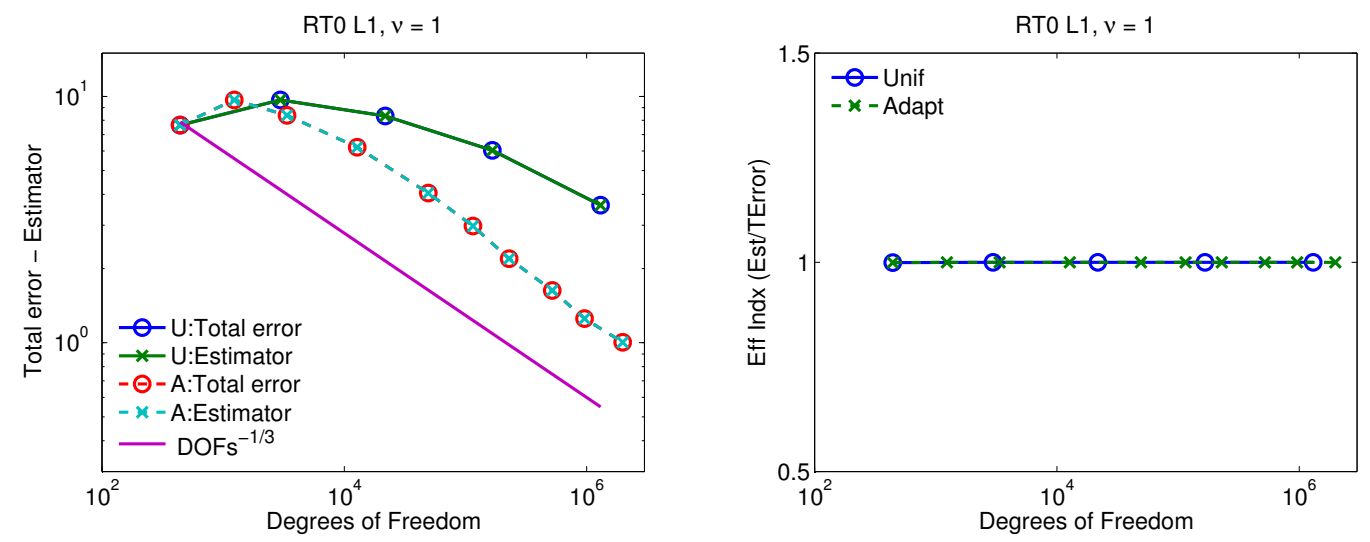

Figure 25: Example 6.4. Decay of total error and estimator (left) and efficiency indices for the adaptive (A) and uniform (U) algorithms.
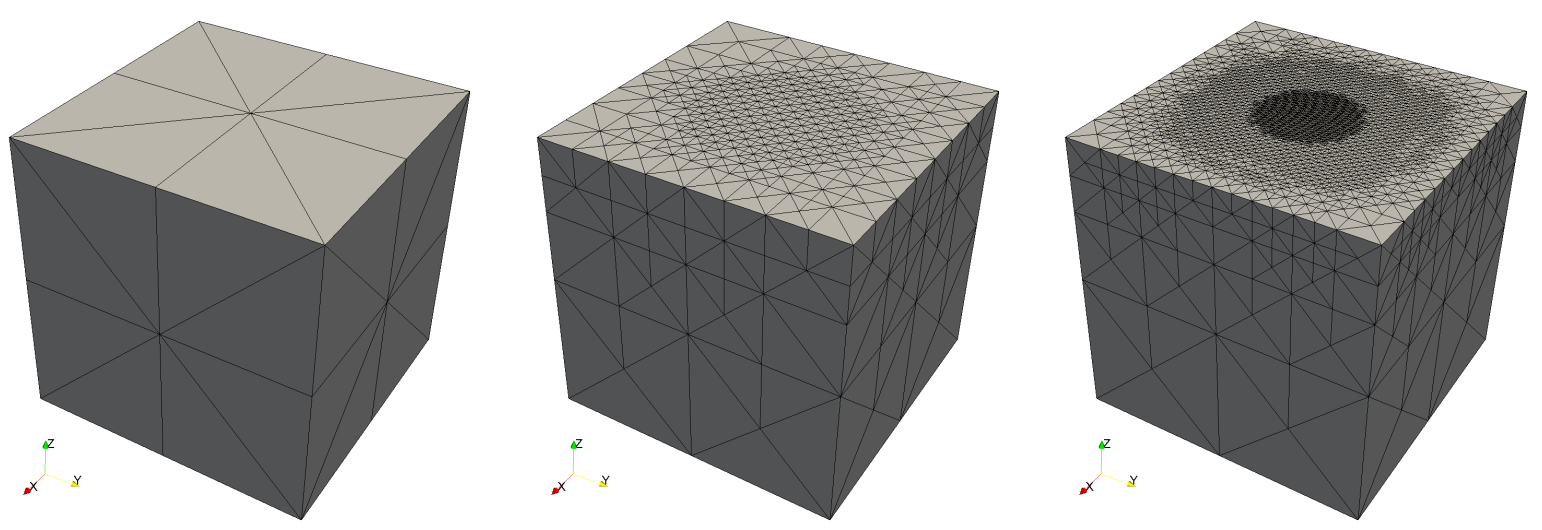

Figure 26: Example 6.4. Meshes generated by the adaptive process at iterations 0,4 and 7 

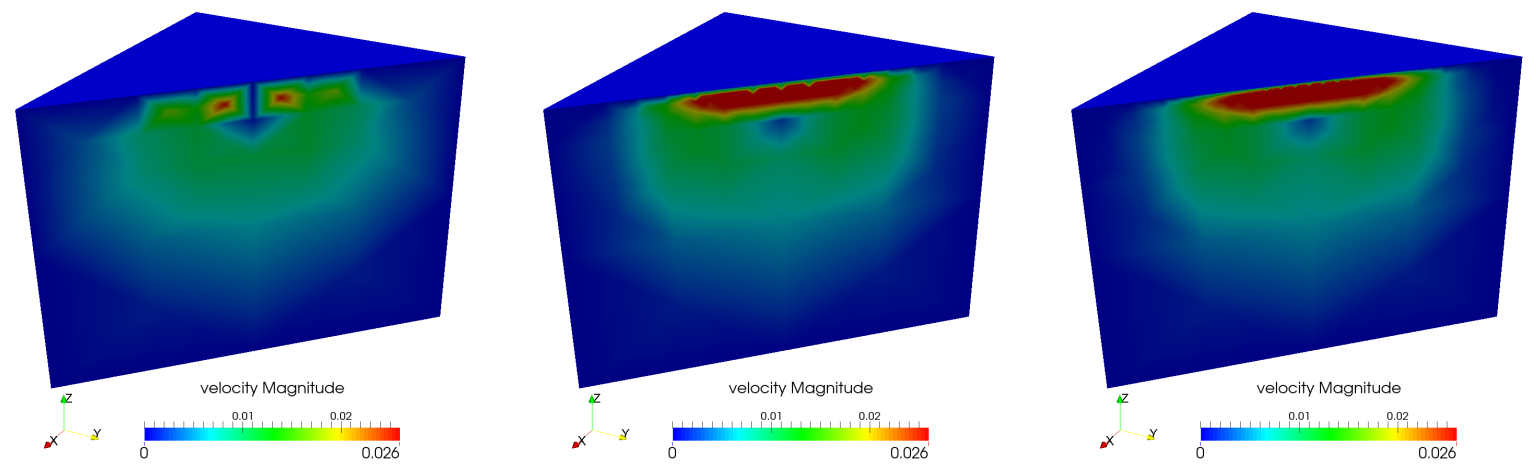

Figure 27: Example 6.4. Module of the velocity field at iterations 3, 6 and 8 of the adaptive process
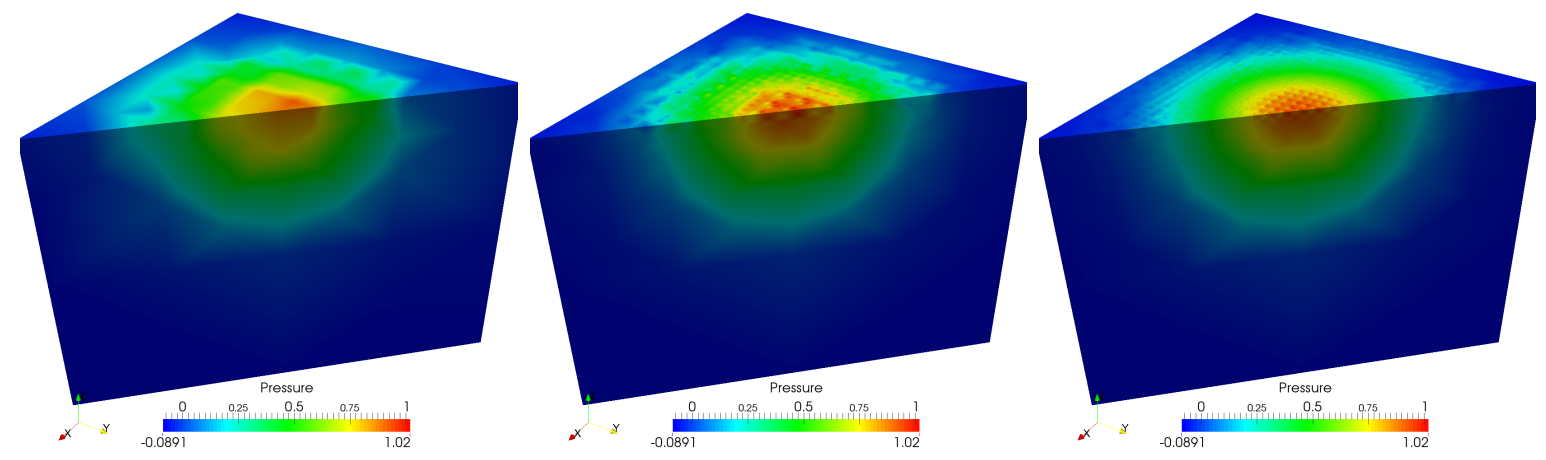

Figure 28: Example 6.4. Pressure at iterations 3,6 and 8 of the adaptive process 


\section{Conclusions}

We proposed and analyzed a new augmented dual-mixed variational formulation for the Oseen problem in the pseudostres-velocity variables. We obtained sufficient conditions on the stabilization parameters such that the augmented variational formulation and the corresponding Galerkin scheme are well-posed and a Céa estimate holds whatever finite element subspaces are used. We also obtained error estimates when Raviart-Thomas or Brezzi-Douglas-Marini elements are used to approximate the rows of the pseudostress, and continuous piecewise polynomials are used to approximate the velocity components. The pressure can be recovered from the pseudostress; in this case, we deduced an a priori estimate in the $L^{2}(\Omega)$-norm (see Remark after Theorem 3).

Moreover, we developed a residual-based a posteriori error analysis and proposed a simple a posteriori error indicator that is shown to be reliable and locally efficient. This a posteriori error indicator consists only of two residual terms, namely, the residual in the momentum equation and the residual in the incompressibility condition. It is valid in two and three dimensions, and for any conforming finite element approximation. We remark that we characterized the behavior of the constants in the a priori error analysis as well as the a posteriori error analysis in terms of the problem parameters (viscosity and advective velocity).

We performed some numerical experiments to support our theoretical results. Numerical results show that the adaptive refinement algorithm performs better than the uniform refinement algorithm concerning the reduction of the total error, in the velocity and pseudostress variables. Moreover, the adaptive algorithm is able to detect boundary and inner layers. We appreciated certain oscillations in the pressure that decrease asymptotically as the mesh is refined. It appears necessary to use some post-processing procedure to recover this variable.

\section{Acknowledgements}

The authors wish to thank the anonymous referees for their valuable comments that help to improve the paper.

\section{References}

[1] M. Ainsworth and J.T. Oden, A posteriori error estimators for the Stokes and Oseen equations, SIAM J. Numer. Anal., vol. 34, pp. 228-245 (1997).

[2] T. Apel, T. Knopp and G. Lube, Stabilized finite element methods with anisotropic mesh refinement for the Oseen problem, Appl. Numer. Math., vol. 58, pp. 1830-1843 (2008).

[3] D.N. Arnold, J. Douglas and Ch.P. Gupta, A family of higher order mixed finite element methods for plane elasticity, Numer. Math., vol. 45, pp. 122 (1984).

[4] G.R. Barrenechea and F. Valentin, A residual local projection method for the Oseen equation, Comput. Methods Appl. Mech. Engrg., vol. 199, pp. 1906-1921 (2010). 
[5] T.P. Barrios, E.M. Behrens and M. González, Low cost a posteriori error estimators for an augmented mixed FEM in linear elasticity, Appl. Numer. Math. 84 (2014) 46-65.

[6] T.P. Barrios, R. Bustinza, G.C. García and E. Hernández, On stabilized mixed methods for generalized Stokes problem based on the velocity-pseudostress formulation: A priori error estimates, Comput. Methods Appl. Mech. Engrg., vol. 237-240 (2012) 78-87.

[7] T.P. Barrios, J.M. Cascón and M. González, A posteriori error analysis of an augmented mixed finite element method for Darcy flow, Comput. Methods Appl. Mech. Engrg., vol. 283 (2015) 909-922.

[8] T.P. Barrios, G.N. Gatica, M. González and N. Heuer, A residual based a posteriori error estimator for an augmented mixed finite element method in linear elasticity, ESAIM: M2AN, vol. 40, pp. 843869 (2006).

[9] R. Becker and M. Braack, A finite element pressure gradient stabilization for the Stokes equations based on local projections, Calcolo 38(4) (2001) 173-199.

[10] M. Braack, E. Burman, V. John and G. Lube, Stabilized finite element methods for the generalized Oseen problem, Comput. Methods Appl. Mech. Engrg., vol. 196, pp. 853-866 (2007).

[11] F. Brezzi and M. Fortin, Mixed and Hybrid Finite Element Methods, Springer-Verlag, 1991.

[12] A.N. Brooks and T.J.R. Hughes, Streamline upwind Petrov-Galerkin formulation for convection dominated flows with particular emphasis on the incompressible Navier-Stokes equations, Comput. Methods Appl. Mech. Engrg. 32 (1982) 199-259.

[13] Z. Cai, C. Tong, P. Vassilevski and C. Wang, Mixed finite element methods for incompressible flow: stationary Stokes equations, Numer. Methods PDEs, 26:4 (2010), 957-978.

[14] Z. Cai, C. Wang and S. Zhang, Mixed finite element methods for incompressible flow: stationary Navier-Stokes equations, SIAM J. Numer. Anal., 48 (2010), 79-94.

[15] R. Codina, Analysis of a stabilized finite element approximation of the Oseen equations using orthogonal subscales, Appl. Numer. Math., vol. 58, pp. 264-283 (2008).

[16] L.P. Franca and S.L. Frey, Stabilized finite element methods: II. The incompressible Navier-Stokes equations, Comput. Methods Appl. Mech. Engrg. 99 (1992) 209-233.

[17] G.N. Gatica, Analysis of a new augmented mixed finite element method for linear elasticity allowing $\mathbb{R T}_{0}-\mathbb{P}_{1}-\mathbb{P}_{0}$ approximations., ESAIM: M2AN Math. Model. Numer. Anal., 40 (2006) 1-28.

[18] G.N.Gatica, An augmented mixed finite element method for linear elasticity with nonhomogeneous Dirichlet conditions, Electron.Trans.Numer.Anal. 26 (2007) 421438.

[19] G.N. Gatica, A. Márquez and S. Meddahi, An augmented mixed finite element method for 3D linear elasticity problems, J. Comput. Appl. Math. 231 (2009) 526540. 
[20] M. González, Stabilized dual-mixed method for the problem of linear elasticity with mixed boundary conditions, Applied Mathematics Letters, 30 (2014) 1-5.

[21] M. González, J. Jansson and S. Korotov, A posteriori error analysis of a stabilized mixed FEM for convection-diffusion problems, Discrete Contin. Dyn. Syst. 2015, Dynamical systems, differential equations and applications. 10th AIMS Conference. Suppl., 525532.

[22] P. Hansbo and A. Szepessy, A velocity-pressure streamline diffusion finite element method for the incompressible Navier-Stokes equations, Comput. Methods Appl. Mech. Engrg. 84 (1990) 175-192.

[23] T.J.R. Hughes, L.P. Franca and M. Balestra, A new finite element formulation for computational fluid dynamics: V. Circumventing the Babuska-Brezzi condition: a stable Petrov-Galerkin formulation of the Stokes problem accommodating equal-order interpolations, Comput. Methods Appl. Mech. Engrg. 59 (1986) 85-99.

[24] C. Johnson and J. Saranen, Streamline diffusion methods for the incompressible Euler and NavierStokes equations, Math. Comp. 47 (1986) 175, 1-18.

[25] A. Masud and T.J.R. Hughes, A stabilized mixed finite element method for Darcy flow, Comput. Methods Appl. Mech. Engrg., vol. 191, pp. 4341-4370 (2002).

[26] J.E. Roberts and J.-M. Thomas, Mixed and Hybrid Methods, in Handbook of Numerical Analysis, edited by P.G. Ciarlet and J.L. Lions, vol. II, Finite Element Methods (Part 1). North-Holland, Amsterdam (1991).

[27] A. Schmidt and K. G. Siebert. Design of Adaptive Finite Element Software: The Finite Element Toolboox ALBERTA, LNCSE 42. Springer (2005).

[28] L. Tobiska and G. Lube, A modified streamline diffusion method for solving the stationary NavierStokes equations, Numer. Math. 59 (1991) 13-29. 